\title{
Quantum Hall effect originated from helical edge states in $\mathrm{Cd}_{3} \mathbf{A s}_{2}$
}

\author{
Rui Chen $\oplus^{1,2}$ C. M. Wang, ${ }^{3,1,4}$ Tianyu Liu, ${ }^{5,1}$ Hai-Zhou Lu, ${ }^{1,4, *}$ and X. C. Xie ${ }^{6,7,8}$ \\ ${ }^{1}$ Shenzhen Institute for Quantum Science and Engineering and Department of Physics, Southern University of Science and Technology \\ (SUSTech), Shenzhen 518055, China \\ ${ }^{2}$ School of Physics, Southeast University, Nanjing 211189, China \\ ${ }^{3}$ Department of Physics, Shanghai Normal University, Shanghai 200234, China \\ ${ }^{4}$ Shenzhen Key Laboratory of Quantum Science and Engineering, Shenzhen 518055, China \\ ${ }^{5}$ Max-Planck-Institut für Physik komplexer Systeme, 01187 Dresden, Germany \\ ${ }^{6}$ International Center for Quantum Materials, School of Physics, Peking University, Beijing 100871, China \\ ${ }^{7}$ Beijing Academy of Quantum Information Sciences, Beijing 100193, China \\ ${ }^{8}$ CAS Center for Excellence in Topological Quantum Computation, University of Chinese Academy of Sciences, Beijing 100190, China
}

(Received 29 May 2021; accepted 23 August 2021; published 9 September 2021)

\begin{abstract}
The recent experimental observations of the quantum Hall effect in three-dimensional (3D) topological semimetals have attracted great attention, but there are still debates on its origin. We systematically study the dependence of the quantum Hall effect in topological semimetals on the thickness, Fermi energy, and growth direction, taking into account the contributions from the Fermi-arc surface states, confinement-induced bulk subbands, and helical side-surface edge states. In particular, we focus on the intensively studied Dirac semimetal $\mathrm{Cd}_{3} \mathrm{As}_{2}$ and its slabs grown along experimentally accessible directions, including [001], [110], and [112]. We reveal an ignored mechanism from the Zeeman splitting of the helical edge states, which along with Fermi-arc 3D quantum Hall effect, may give a nonmonotonic dependence of the Hall conductance plateaus on the magnetic field in the most experimentally studied [112] direction slab. Our results will be insightful for exploring the quantum Hall effects beyond two dimensions.
\end{abstract}

DOI: 10.1103/PhysRevResearch.3.033227

\section{INTRODUCTION}

Since the discovery of the quantum Hall effect in twodimensional (2D) electron gases [1,2], tremendous efforts have been devoted to generalizing the exotic phase of matter to higher dimensions [3-13], in the absence of magnetic fields $[14,15]$, or the nonlinear-response regime [16-21]. Recently, quantized Hall conductance plateaus were observed in the topological Dirac semimetal $\mathrm{Cd}_{3} \mathrm{As}_{2}$, with sample thickness ranging from tens to hundreds of nanometers [22-30]. One of the mechanisms is a three-dimensional (3D) quantum Hall effect supported by the Fermi-arc surface states in the topological semimetal [6-8], which also can support a quantum oscillation [31-34]. Nevertheless, the nature of the quantum Hall effect in 3D topological semimetals is still in debate (Table I) and has been attracting growing attention.

In this work, we report a new mechanism of the quantum Hall effect in topological semimetals. We numerically calculate the Hall conductance of the Dirac-semimetal $\mathrm{Cd}_{3} \mathrm{As}_{2}$ slabs grown along three experimentally accessible and widely

\footnotetext{
*Corresponding author: luhz@sustech.edu.cn

Published by the American Physical Society under the terms of the Creative Commons Attribution 4.0 International license. Further distribution of this work must maintain attribution to the author(s) and the published article's title, journal citation, and DOI.
}

investigated crystallographic directions. For the slab grown along the [001] direction, the magnitude of the quantized Hall conductance increases with the increasing magnetic field, as a result of the Zeeman splitting of the helical edge states on the side surfaces (Fig. 1). The mechanism was previously ignored and originated from the nontrivial topology of the confinement induced bulk subbands characterized by the spin Chern number. In contrast, for the slab grown along the [110] direction, the Hall plateaus decrease with the increasing magnetic field, due to the Fermi-arc 3D quantum Hall effect [6]. As a result, the Hall conductance in the slab grown along the [112] direction can be understood as a competition between the helical edge states and Fermi-arc surface states, with Hall plateaus decreasing in the weak-field region but growing in the strong-field region.

\section{MODEL AND METHOD}

We start with an effective Hamiltonian for the Dirac semimetal $\mathrm{Cd}_{3} \mathrm{As}_{2}$ [35], which reads

$$
H=\varepsilon_{0}(\mathbf{k})+\left[\begin{array}{cccc}
M(\mathbf{k}) & A k_{+} & 0 & 0 \\
A k_{-} & -M(\mathbf{k}) & 0 & 0 \\
0 & 0 & M(\mathbf{k}) & -A k_{-} \\
0 & 0 & -A k_{+} & -M(\mathbf{k})
\end{array}\right]
$$

where $k_{ \pm}=k_{x} \pm i k_{y}, \varepsilon_{0}(\mathbf{k})=C_{0}+C_{1} k_{z}^{2}+C_{2}\left(k_{x}^{2}+k_{y}^{2}\right)$, and $M(\mathbf{k})=M_{0}+M_{1} k_{z}^{2}+M_{2}\left(k_{x}^{2}+k_{y}^{2}\right)$. The $x, y$, and $z$ axes in the Hamiltonian are defined along the [100], [010], and [001] 
TABLE I. The slab growth direction, thickness, and explanation in the recent experiments on the quantum Hall effect in the topological semimetal $\mathrm{Cd}_{3} \mathrm{As}_{2}$.

\begin{tabular}{|c|c|c|c|}
\hline Refs. & Direction & Thickness (nm) & Explanation in Refs. \\
\hline$[32]$ & [010] & $150-2000$ & Weyl orbit \\
\hline$[30]$ & [001] & $45-50$ & $\begin{array}{l}\text { Topological insulator } \\
\text { type surface states }\end{array}$ \\
\hline$[22],[23]$ & [112] & $55-71,80-150$ & Weyl orbit \\
\hline$[24],[25]$ & [112] & 80,100 & Mixed Fermi arcs \\
\hline$[26],[27]$ & [112] & $20,38-43$ & Surface states \\
\hline$[28],[29]$ & [112] & $12-23,35$ & Bulk subbands \\
\hline
\end{tabular}

crystallographic directions, respectively. The model hosts two pairs of Weyl nodes at $\mathbf{k}=\left(0,0, \pm k_{\mathrm{w}}\right)$ with the energy $E_{\mathrm{w}}=$ $C_{0}-C_{1} M_{0} / M_{1}$ and $k_{\mathrm{w}}=\sqrt{\left|M_{0} / M_{1}\right|}$. We take the parameters for $\mathrm{Cd}_{3} \mathrm{As}_{2}$ as $C_{0}=-0.0145 \mathrm{eV}, C_{1}=10.59 \mathrm{eV}^{2}, C_{2}=$ $11.5 \mathrm{eVA} \AA^{2}, M_{0}=0.0205 \mathrm{eV}, M_{1}=-18.77 \mathrm{eV}^{2}, M_{2}=$ $-13.5 \mathrm{eV} \AA^{2}, A=0.889 \mathrm{eV} \AA$ [36]. The samples of $\mathrm{Cd}_{3} \mathrm{As}_{2}$ are usually cleaved or grown along the [110] [37], [001] [30], or [112] directions [24,29]. To obtain the dispersion of the Dirac semimetal slab along an arbitrary growth direction, we rotate the $y$ axis to the $y^{\prime}$ axis through the rotation matrix [6]

$$
\left(\begin{array}{l}
k_{x}^{\prime} \\
k_{y}^{\prime} \\
k_{z}^{\prime}
\end{array}\right)=\left(\begin{array}{ccc}
\cos \alpha & \sin \alpha & 0 \\
-\cos \theta \sin \alpha & \cos \theta \cos \alpha & \sin \theta \\
\sin \theta \sin \alpha & -\sin \theta \cos \alpha & \cos \theta
\end{array}\right)\left(\begin{array}{l}
k_{x} \\
k_{y} \\
k_{z}
\end{array}\right) .
$$
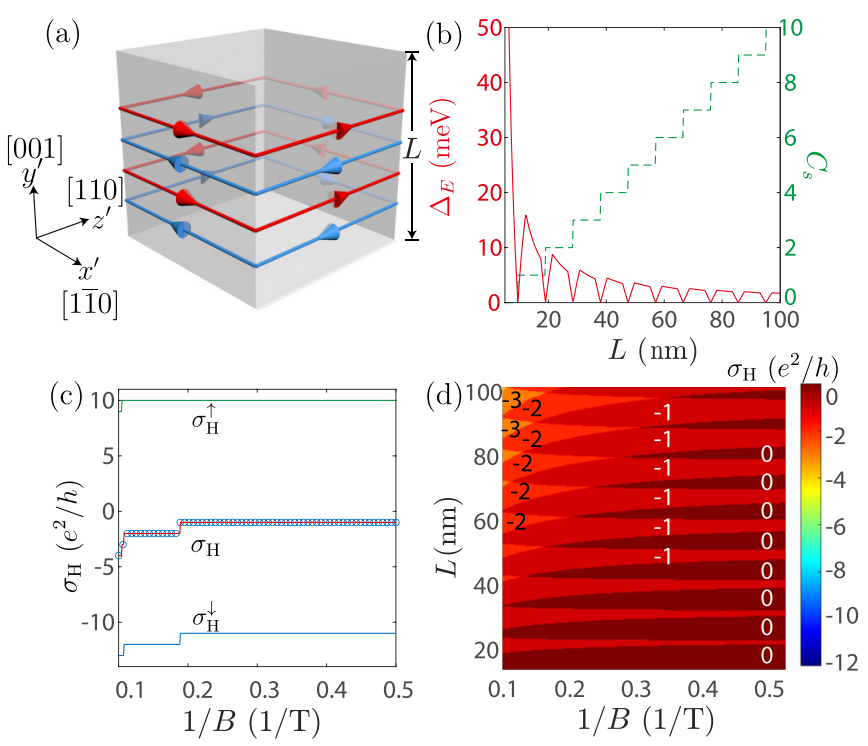

FIG. 1. The [001]-direction slab of the Dirac semimetal. (a) The helical edge states on the side surfaces in the $\left(x^{\prime}, y^{\prime}, z^{\prime}\right)$ coordinates. The arrows denote the directions of propagation, and the red and blue colors distinguish opposite spin polarizations. (b) The confinementinduced energy gap $\Delta_{E}$ (red solid line) and the spin Chern number $C_{s}$ (green dashed line) as functions of the slab thickness $L$. (c) The Hall conductance $\sigma_{\mathrm{H}}$ (blue dots) and $\sigma_{\mathrm{H}}^{\uparrow, \downarrow}$ (blue and green lines) as functions of the magnetic field $1 / B$ for $L=100 \mathrm{~nm}$. The red line correspond to $\sigma_{\mathrm{H}}^{\uparrow}+\sigma_{\mathrm{H}}^{\downarrow}$. (d) $\sigma_{\mathrm{H}}$ as a function of $L$ and $1 / B$. The Fermi energy $E_{\mathrm{F}}$ is at the Weyl node $E_{\mathrm{w}}$.
The [110], [112], and [001] directions correspond to $(\theta, \alpha)=$ $(0,-\pi / 4),(\arctan \sqrt{2},-\pi / 4)$, and $(\pi / 2,-\pi / 4)$, respectively. We include a magnetic field always normal to the cleave surface $\mathbf{B}=(0, B, 0)$. The Zeeman term has the form $H_{\text {Zeeman }}=\frac{\mu_{B}}{2}(\boldsymbol{\sigma} \cdot \mathbf{B}) \otimes \frac{1}{2}\left[g_{s}\left(\sigma_{0}+\sigma_{z}\right)+g_{p}\left(\sigma_{0}-\sigma_{z}\right)\right]$, where $\mu_{B}$ is the Bohr magneton, $g_{s}=18.6$ and $g_{p}=2$ are the $g$ factors [38].

The Hall conductance for a slab of thickness $L$ can be found as $\sigma_{\mathrm{H}}=\sigma L$, where the Hall conductivity can be found from the Kubo formula [39]

$$
\sigma=\frac{e^{2} \hbar}{i V_{\text {eff }}} \sum_{\delta^{\prime} \neq \delta} \frac{\left\langle\Psi_{\delta}\left|v_{x}\right| \Psi_{\delta^{\prime}}\right\rangle\left\langle\Psi_{\delta^{\prime}}\left|v_{z}\right| \Psi_{\delta}\right\rangle\left[f\left(E_{\delta}\right)-f\left(E_{\delta^{\prime}}\right)\right]}{\left(E_{\delta}-E_{\delta^{\prime}}\right)\left(E_{\delta}-E_{\delta^{\prime}}+i \Gamma\right)},
$$

where $e$ is the elementary charge, $\hbar$ is the reduced Planck constant, $V_{\text {eff }}$ is the volume of the slab, $\left|\Psi_{\delta}\right\rangle$ is the eigenstate of energy $E_{\delta}$ for $H$ in the $y^{\prime}$-direction magnetic field and open boundaries at $y^{\prime}= \pm L / 2, v_{x}$ and $v_{z}$ are the velocity operators, $f(x)$ is the Fermi distribution.

\section{SLABS GROWN ALONG DIFFERENT DIRECTIONS}

\section{A. [001] slab}

For a $\mathrm{Cd}_{3} \mathrm{As}_{2}$ slab grown along the [001] direction, the bulk spectrum is quantized into discrete gapped subbands (see Appendix A) because of the quantum confinement effect [40-42]. The spectrum opens a gap, which decays with increasing $L$ (probably with an oscillation as well). The effective Hamiltonian $\mathcal{H}_{n n}$ for each subband $n(=1,2, \ldots)$ is equivalent to a quantum spin Hall insulator [43-45] characterized by the spin Chern number [46-48] $C_{s}^{n}=\left(C_{n}^{\uparrow}-C_{n}^{\downarrow}\right) / 2$, where $C_{n}^{\uparrow, \downarrow}= \pm \frac{1}{2}\left[\operatorname{sgn}\left(M_{0}+M_{1} n^{2} \pi^{2} / L^{2}\right)-\operatorname{sgn}\left(M_{2}\right)\right][46]$ are the valence-band Chern numbers of the spin-up and spin-down blocks of the $n$th subband (see Appendix B). Each Chern number $C_{n}^{\uparrow / \downarrow}$ represents a chiral edge state circulating around the side surfaces [Fig. 1(a)]. The total spin Chern number $C_{s}=\sum_{n} C_{s}^{n}$ is equal to the number of pairs of helical edge states. As shown in Fig. 1(b), the oscillatory decay of the band gap with increasing $L$ is always accompanied by the variation of the spin Chern number $C_{s}$ at each dip. In the Dirac semimetal $\mathrm{Na}_{3} \mathrm{Bi}$, a topological phase transition to the quantum spin Hall state has been observed [49].

However, the spin Chern number is not measurable because the measurable Hall conductance is associated with the total Chern number $\sigma_{\mathrm{H}}=\frac{e^{2}}{h} \sum_{n, s=\uparrow, \downarrow} C_{n}^{s}$, which is zero in the absence of the magnetic field because of time-reversal symmetry. A magnetic field can break time-reversal symmetry as well as the balance between $C_{n}^{\uparrow}$ and $C_{n}^{\downarrow}$, leading to measurable Hall conductance $\sigma_{\mathrm{H}}$ whose magnitude increases with increasing magnetic field, as shown in Figs. 1(c) and 1(d). We also plot the Hall conductance $\sigma_{\mathrm{H}}^{\uparrow, \downarrow}$ for the spin-up and spin-down blocks of the Hamiltonian [Fig. 1(c)], which confirms that the nonzero quantum Hall conductance is originated from the field-induced imbalance between counterpropagating chiral edge states. Also, Fig. 1(d) shows that the Hall conductance approaches zero for thinner slabs because of the mixing of counterpropagating chiral edge states. This mechanism due to the splitting of helical edge states was previously ignored and could benefit further experimental explorations. 
(a)

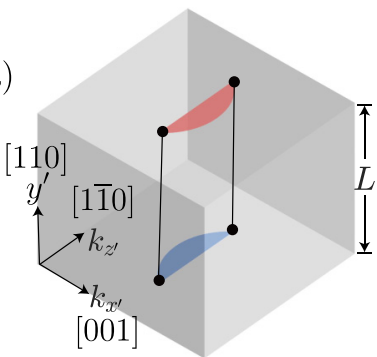

(c)

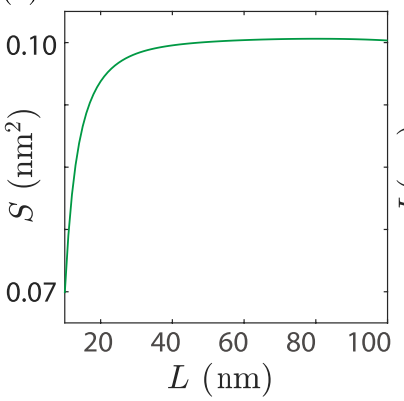

(d)

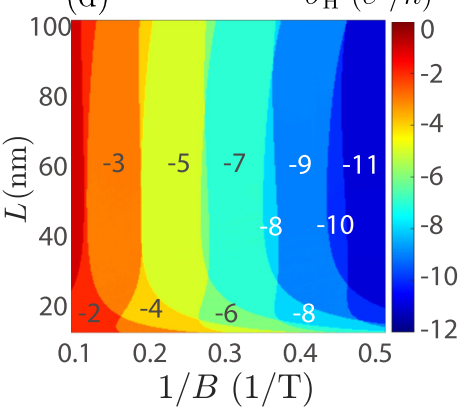

(b)

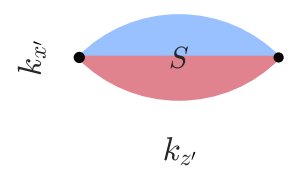

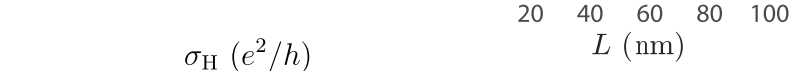

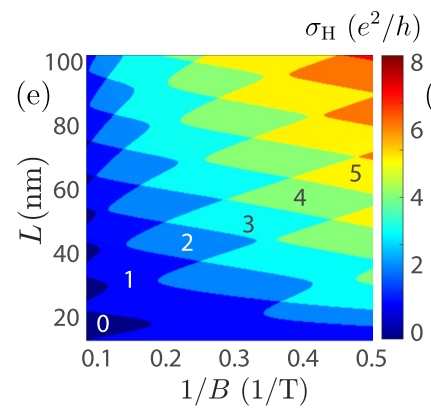

(c)

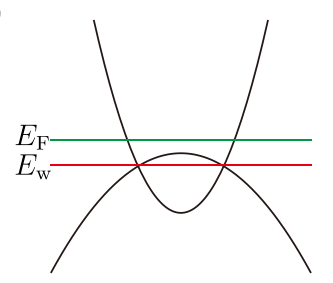

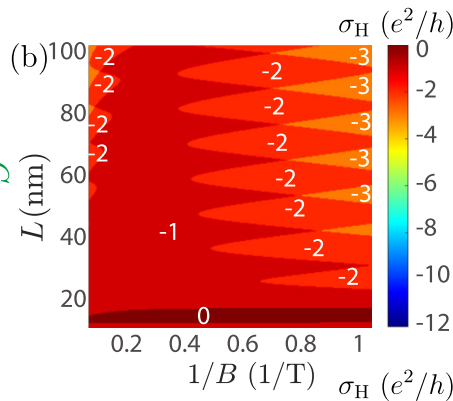

(d) 1

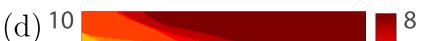

(a)
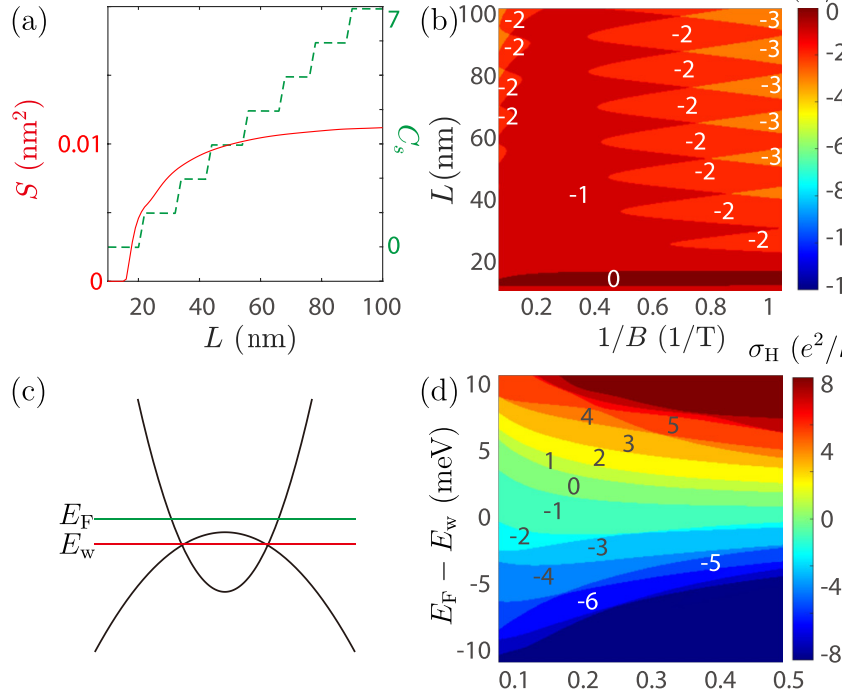

FIG. 2. The [110]-direction slab of the Dirac semimetal, which consists of a Weyl semimetal and its time reversal. (a) The real-space correspondence of Fermi-arc surface states (red for top and blue for bottom) of the Weyl semimetal described by the upper block of $H$ in Eq. (1), for a slab with open boundary condition along the $y^{\prime}$ direction. $E_{\mathrm{w}}$ marks the Weyl-node energy. (b) The projection of the Fermi arc on the $k_{x^{\prime}}-k_{z^{\prime}}$ plane. $S$ is the area of the Fermi loop. (c) The area $S$ of the Fermi surface of the Fermi-arc surface states in the $\left(k_{x^{\prime}}, y^{\prime}, k_{z^{\prime}}\right)$ plane as a function of the slab thickness $L$. (d) The Hall conductance $\sigma_{\mathrm{H}}$ as a function of $L$ and magnetic field $1 / B$. The Fermi energy $E_{\mathrm{F}}$ is at the Weyl node $E_{\mathrm{w}}$.

\section{B. [110] slab}

In contrast to the above [001] case, for a semimetal slab grown along the [110] and equivalent [100] or [011] direction, there exists a 3D quantum Hall effect $[6,13]$ arising from the Fermi-arc surface states $[31,33]$. On each of the top and bottom surfaces of a Weyl semimetal, there are topologically protected surface states, which can be regarded as half of a 2D electron gas. Their open Fermi surfaces are dubbed as the Fermi arcs. The 2D Fermi-arc surface states on opposite surfaces can be connected through the bulk states to form a complete 2D electron gas with a 3D spatial distribution and closed Fermi surface [Figs. 2(a) to 2(c), red for top and blue for bottom surfaces], to support a "3D" quantum Hall effect [6]. A Dirac semimetal can be regarded as two time-reversed Weyl semimetals to host two copies of the Fermi-arc quantum Hall effect. In addition, in a Dirac semimetal, the Fermi-arc surface states and their time-reversal partners on a single surface can form a 2D electron gas [50], to support a quantum Hall effect as well. For both cases, the Hall conductance plateaus are supposed to decrease with increasing magnetic field, much like those in conventional 2D electron gases [1], where the magnetic field presses the occupation of electrons to lower Landau levels, as shown in Fig. 2(d) for different slab thickness $L$.

Moreover, Fig. 2(d) shows that the width of the quantized plateaus is stable for thicker slabs $(L>50 \mathrm{~nm})$, while show

FIG. 3. The [112]-direction slab of the Dirac semimetal. (a) The area of the Fermi surface $S$ (solid) and spin Chern number $C_{s}$ (dotted) as functions of the thickness $L$. (b) The Hall conductance $\sigma_{\mathrm{H}}$ as a function of $L$ and magnetic field $1 / B$. (c) Schematic of where the Fermi energy $E_{\mathrm{F}}$ and Weyl modes $E_{\mathrm{w}}$ are in the bulk spectrum of the Dirac semimetal. (d)-(f) $\sigma_{\mathrm{H}}$ as a function of (d) $\left(E_{\mathrm{F}}-E_{\mathrm{w}}, 1 / B\right)$ and (e,f) $(L, 1 / B)$, respectively. Here we take (d) $L=50 \mathrm{~nm}$, (e) $E_{\mathrm{F}}-$ $E_{\mathrm{w}}=5 \mathrm{meV}$, and (f) $E_{\mathrm{F}}-E_{\mathrm{w}}=-5 \mathrm{meV}$.

obvious variations for ultrathin slabs $(L<20 \mathrm{~nm})$ with decreasing thickness. This can be understood using Figs. 2(b) and 2(c), where the area of the Fermi loop $S$ converges to a constant for thick slabs, but decreases exponentially with decreasing $L$ due to the hybridization of the opposite surfaces in ultrathin slabs. According to the Lifshitz-Onsager relation, $S$ determines the plateau width of the Hall conductance, which explains the quantized pattern in Fig. 2(d). Moreover, in Dirac semimetals $E_{\mathrm{F}}$ shifts away from $E_{\mathrm{w}}$ as the Zeeman effect splits $E_{\mathrm{w}}$ (see Appendix C), leading to a systematic shift of the Hall plateaus with increasing thickness [Fig. 2(d)].

\section{C. [112] slab}

The slabs along the [112] direction inherits the properties of both the confinement-induced helical edge states in the [001] slab and Fermi-arc surface states in the [110] slab. Figure 3(b) shows the Hall conductance of the slab along the [112] direction as a function of $1 / B$ for different $L$. For weak magnetic fields $[1 / B>0.4(1 / \mathrm{T})]$, the oscillation pattern of the quantized Hall conductance is similar to that of the [110] slab, that is, the Hall plateaus decrease with increasing 

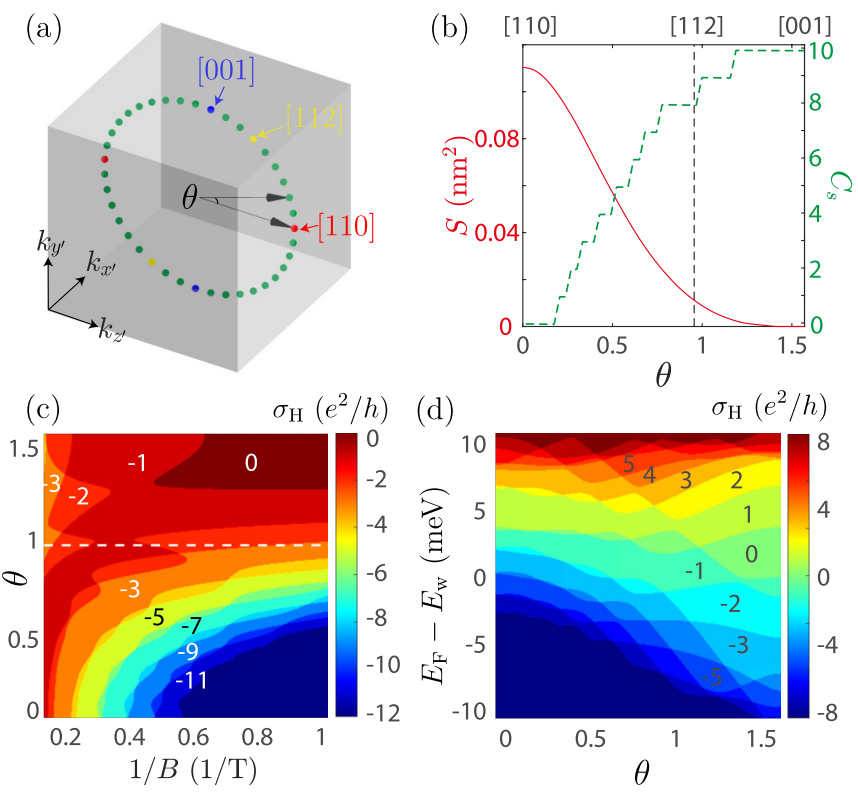

FIG. 4. The dependence on $\theta$, the angle between the line connecting the Dirac nodes and $k_{x^{\prime}}-k_{z^{\prime}}$ plane. (a) Illustration of $\theta$. (b) The area of the Fermi surface of the Fermi-arc surface states $S$ (solid) and spin Chern number $C_{s}$ (dotted) as functions of $\theta$. (c,d) The Hall conductance $\sigma_{\mathrm{H}}$ as a function of $(\theta, 1 / B)$ and $\left(E_{\mathrm{F}}-E_{\mathrm{w}}, 1 / B\right)$, respectively. The dashed line corresponds to the slab grown along the [112] direction. Here we take (c) $E_{\mathrm{F}}-E_{\mathrm{w}}=0$ and (d) $B=5 \mathrm{~T}$.

magnetic field, which indicates the quantized conductance is mainly originated from the Fermi-arc surface states. Compared to the [110] slab, the width of the plateaus is larger because of the smaller area enclosed by the Fermi arc $S$ [Fig. 3(a)]. For strong magnetic fields $[1 / B<0.4(1 / \mathrm{T})]$, the Hall conductance increases with increasing magnetic field, similar to that in the [001] slab, indicating that it is mainly contributed to by the imbalance of the helical edge states.

Above, the Fermi energy is assumed to cross the Weyl nodes, i.e., $E_{\mathrm{F}}=E_{\mathrm{w}}$ [see Fig. 3(c) for the definitions of $E_{\mathrm{F}}$ and $\left.E_{\mathrm{w}}\right]$. Figure $3(\mathrm{~d})$ shows $\sigma_{\mathrm{H}}$ in the $\left(E_{\mathrm{F}}-E_{\mathrm{W}}, 1 / B\right)$ plane with $L=50 \mathrm{~nm}$. For the Fermi energies far away from the Weyl nodes, the quantum Hall effect originates from the confinement-induced bulk subbands [Figs. 3(e) and 3(f)], and the Hall conductance monotonically decreases with increasing magnetic field for different thickness and Fermi energies, different from the non-monotonic dependence when $E_{\mathrm{F}}=E_{\mathrm{W}}$ or small $E_{F}-E_{w}$.

\section{Angle dependence}

Figure 4(a) illustrates the dependence on $\theta$, the angle between the line connecting the Dirac nodes and the $k_{x^{\prime}}-k_{z^{\prime}}$ plane. For example, the Dirac nodes are located on the $k_{x^{\prime}}-k_{z^{\prime}}$ plane when $\theta=0$ [Figs. 2(a) and 2(b)]. Figure 4(b) shows that $S$ decreases and $C_{s}$ increases with increasing $\theta$, indicating the competition between the Fermi-arc surface states and sidesurface helical edge states. Figure 4(c) shows $\sigma_{\mathrm{H}}$ as a function of $\theta$ and $1 / B$ for $L=100 \mathrm{~nm}$. For $\theta=0$ ([110] direction) and $\theta=\pi / 2$ ([001] direction), the quantized conductance is only contributed by the Fermi-arc surface states and imbalanced
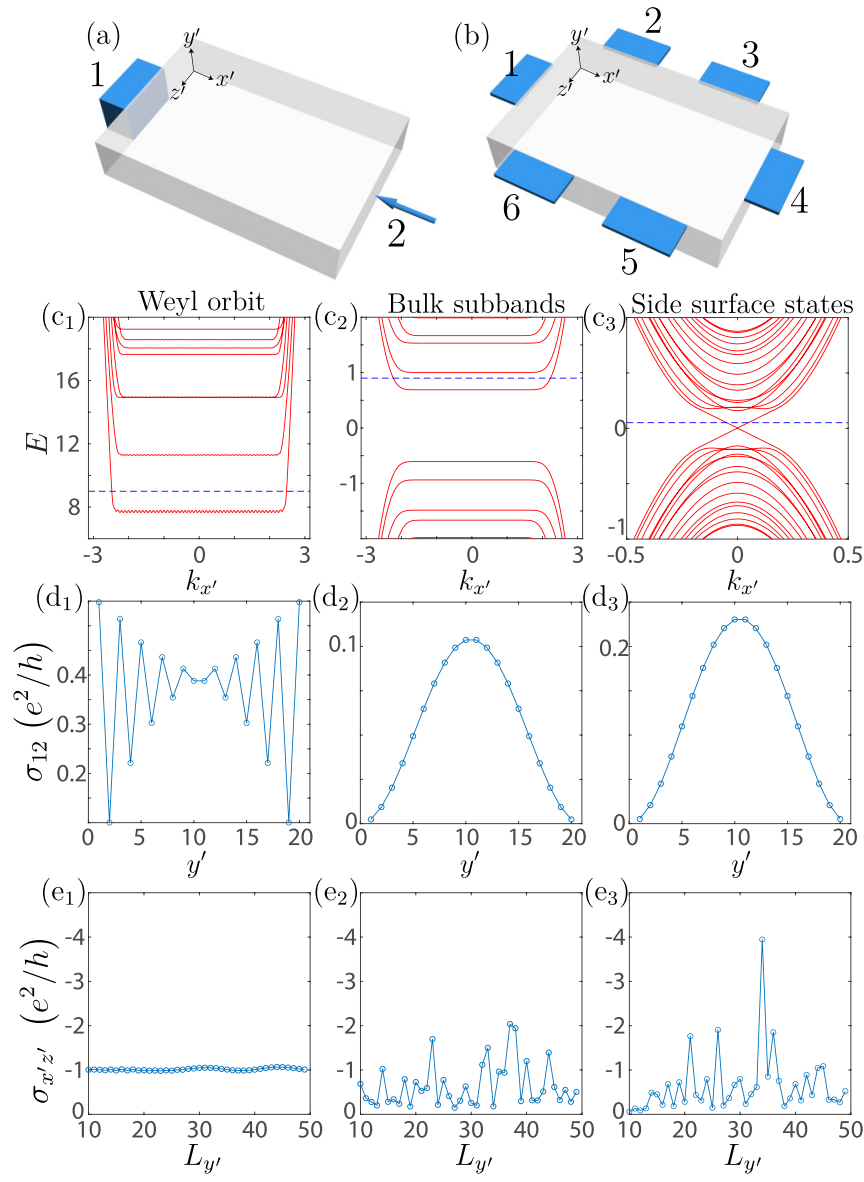

FIG. 5. (a) Schematic illustration of the 3D device with a electrode attached on the left boundary, and the right lead is scanned by the STM tip as a 1D normal lead. (b) Schematic illustration of the 3D device with six electrodes on the top surface. $\left(c_{1}\right)-\left(c_{3}\right)$ The spectra for the three different cases that possess the quantized Hall conductance. Here the blue dashed lines label the Fermi energy. $\left(d_{1}\right)-\left(d_{3}\right)$ and $\left(e_{1}\right)-\left(e_{3}\right)$ correspond to the numerically calculated two-terminal conductance $\sigma_{12}$ and the surface Hall conductance $\sigma_{x^{\prime} z^{\prime}}$ by using the devices shown in (a) and (b), respectively.

helical edge states, respectively. For other $\theta$, the quantized Hall conductance originates from both the Fermi-arc surface states and the helical edge states. Furthermore, $\sigma_{\mathrm{H}}$ as a function of $\left(E_{\mathrm{F}}-E_{\mathrm{w}}, \theta\right)$ [Fig. 4(d)] shows that the quantum Hall effect may be due to the confinement-induced bulk subbands, giving another origin for the experimentally observed quantum Hall effect in $\mathrm{Cd}_{3} \mathrm{As}_{2}$ [28,29]. In experiments, it may be difficult to distinguish whether the thickness-dependent conductance plateaus are consequences of the bulk subbands or the Fermi-arc surface states. The nonmonotonic dependence of $\sigma_{\mathrm{H}}$ on the magnetic field may play another significant role to detect the side-surface helical edge states or Fermi-arc 3D quantum Hall effect in a [112] Dirac semimetal slab.

\section{EXPERIMENTAL PROPOSALS}

Above, we showed that the Hall plateaus can be attributed to the Fermi-arc surface states, confinement-induced bulk subbands, and helical side-surface edge states. It may be 
challenging to distinguish them in a standard Hall-bar device. We propose that the Fermi-arc quantum Hall effect can be identified through the transport setups shown in Figs. 5(a) and 5(b). As shown in Figs. 5( $\left.c_{1}\right)$ to 5( $\left.c_{3}\right)$, we consider the quantum Hall effect originated by three mechanisms: the Weyl orbit, bulk subbands, and the side surface states.

For the two-terminal device [Fig. 5(a)], the two-terminal conductance $\sigma_{12}$ shows maximum values near the top and bottom surfaces for the case of the Weyl orbit [Fig. $5\left(\mathrm{~d}_{1}\right)$ ], and shows maximum values in the central region for the cases of the confinement-induced subbands or the side surface states [Figs. $5\left(\mathrm{~d}_{2}\right)$ and $5\left(\mathrm{~d}_{3}\right)$ ]. This phenomenon can be explained by calculating the wave-function distribution of the edge states between the Landau levels (see Appendix D).

We also calculate the surface Hall conductance by using the device shown in Fig. 5(b). For the case of the Weyl orbit [Fig. 5(e $\left.\mathrm{e}_{1}\right)$ ], the surface Hall conductance $\sigma_{x^{\prime} z^{\prime}}$ is quantized and stable with increasing film thickness $L_{y^{\prime}}$. While for the rest of the cases, $\sigma_{x^{\prime} z^{\prime}}$ is not quantized, and shows obvious fluctuations. Moreover, the quantum Hall effect induced by the bulk subbands and the helical edge states can be identified by measuring the nonlocal bulk transport [51,52]. Therefore, the devices can be used to identify the originations of the observed quantum Hall effect in experiments.

\section{ACKNOWLEDGMENTS}

We thank Hua Jiang, Donghui $\mathrm{Xu}$, and Bin Zhou for helpful discussions. This work was supported by the National Natural Science Foundation of China (Grants No. 11925402 and No. 11974249), the National Basic Research Program of China (Grant No. 2015CB921102), the Strategic Priority Research Program of Chinese Academy of Sciences (Grant No. XDB28000000), the Natural Science Foundation of Shanghai (Grant No. 19ZR1437300), Guangdong province (Grants No. 2020KCXTD001 and No. 2016ZT06D348), Shenzhen High-level Special Fund (Grants No. G02206304 and No. G02206404), and the Science, Technology and Innovation Commission of Shenzhen Municipality (Grants No. ZDSYS20170303165926217, No. JCYJ20170412152620376, and No. KYTDPT20181011104202253). R.C. acknowledges support from the project funded by the China Postdoctoral Science Foundation (Grant No. 2019M661678) and the SUSTech Presidential Postdoctoral Fellowship. The numerical calculations were supported by Center for Computational Science and Engineering of SUSTech.

\section{APPENDIX A: MODEL AND SPECTRUM}

\section{Dirac semimetal slab with open boundary condition along the $y^{\prime}$ direction}

To obtain the Hamiltonian of the Dirac semimetal slab along an arbitrary growth direction, we include the new wave vector $\left(k_{x}^{\prime}, k_{y}^{\prime}, k_{z}^{\prime}\right)$ through the rotation transformation in Eq. (2). We consider the open boundary condition along the $y^{\prime}$ direction with the thickness $L$. In this case, $k_{x}^{\prime}$ and $k_{z}^{\prime}$ are still good quantum numbers while $k_{y}^{\prime}$ should be replaced by $k_{y}^{\prime}=-i \partial_{y^{\prime}}$. The Hamiltonian $H\left(k_{x}^{\prime},-i \partial_{y^{\prime}}, k_{z}^{\prime}\right)$ has the following form:

$$
H=X_{1}+X_{2} \partial_{y^{\prime}}+X_{3} \partial_{y^{\prime}}^{2},
$$

where

$$
\begin{aligned}
& X_{1}=\varepsilon^{\prime}\left(\mathbf{k}^{\prime}\right)+\left(\begin{array}{cccc}
M^{\prime}\left(\mathbf{k}^{\prime}\right) & A e^{i \alpha}\left(k_{x}^{\prime}-i k_{z}^{\prime} \sin \theta\right) & 0 & 0 \\
A e^{-i \alpha}\left(k_{x}^{\prime}+i k_{z}^{\prime} \sin \theta\right) & -M^{\prime}\left(\mathbf{k}^{\prime}\right) & 0 & 0 \\
0 & 0 & M^{\prime}\left(\mathbf{k}^{\prime}\right) & -A e^{-i \alpha}\left(k_{x}^{\prime}+i k_{z}^{\prime} \sin \theta\right) \\
0 & 0 & -A e^{i \alpha}\left(k_{x}^{\prime}-i k_{z}^{\prime} \sin \theta\right) & -M^{\prime}\left(\mathbf{k}^{\prime}\right)
\end{array}\right) \text {, } \\
& X_{2}=-i k_{z}\left(C_{1}-C_{2}\right) \sin 2 \theta+\left(\begin{array}{cccc}
Z_{1} & A e^{i \alpha} \cos \theta & 0 & 0 \\
-A e^{-i \alpha} \cos \theta & -Z_{1} & 0 & 0 \\
0 & 0 & Z_{1} & A e^{-i \alpha} \cos \theta \\
0 & 0 & -A e^{i \alpha} \cos \theta & -Z_{1}
\end{array}\right) \\
& X_{3}=-C_{1} \sin ^{2} \theta-C_{2} \cos ^{2} \theta+\left(\begin{array}{cccc}
Z_{2} & 0 & 0 & 0 \\
0 & -Z_{2} & 0 & 0 \\
0 & 0 & Z_{2} & 0 \\
0 & 0 & 0 & -Z_{2}
\end{array}\right) \text {, }
\end{aligned}
$$

with

$$
\begin{gathered}
\varepsilon^{\prime}\left(\mathbf{k}^{\prime}\right)=C_{0}+C_{1} k_{z}^{\prime 2} \cos ^{2} \theta+C_{2}\left(k_{x}^{\prime 2}+k_{z}^{\prime 2} \sin ^{2} \theta\right) \\
M^{\prime}\left(\mathbf{k}^{\prime}\right)=M_{0}+M_{1} k_{z}^{\prime 2} \cos ^{2} \theta+M_{2}\left(k_{x}^{\prime 2}+k_{z}^{\prime 2} \sin ^{2} \theta\right), \\
Z_{1}=-i k_{z}^{\prime}\left(M_{1}-M_{2}\right) \sin 2 \theta \\
Z_{2}=-M_{1} \sin ^{2} \theta-M_{2} \cos ^{2} \theta .
\end{gathered}
$$



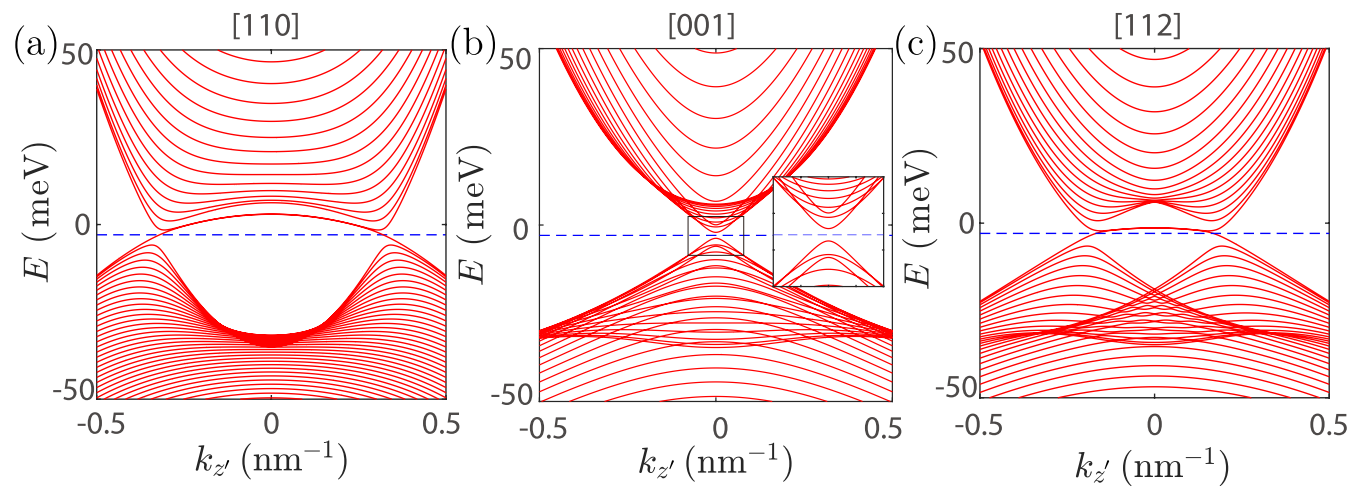

FIG. 6. Numerically calculated spectrum of the Dirac semimetal slab with open boundary condition along the $y^{\prime}$ direction at $k_{x}^{\prime}=0$. The blue dashed lines label the Fermi level $E_{\mathrm{F}}=E_{\mathrm{w}}$. In (a), (b), and (c), $y^{\prime}$ correspond to [110], [001], and [112]-directions, respectively.

Using the basis

$$
\left|\varphi_{n}\left(y^{\prime}\right)\right\rangle=\sqrt{\frac{2}{L}} \sin \left[\frac{n \pi}{L}\left(y^{\prime}+\frac{L}{2}\right)\right]
$$

with $n=1,2,3, \ldots$, the matrix elements are

$$
\mathcal{H}_{m n}=\left\langle\varphi_{m}|H| \varphi_{n}\right\rangle= \begin{cases}X_{1}-\frac{n^{2} \pi^{2}}{L^{2}} X_{3}, & m=n, \\ -\frac{2\left[-1+(-1)^{n+m}\right] n m}{L\left(n^{2}-m^{2}\right)} X_{2}, & m \neq n .\end{cases}
$$

From the above Hamiltonian, we can obtain the eigenenergy (shown in Fig. 6) $E_{\delta}$ and the corresponding eigenvector $C_{\delta, s n}$, where $s=1,2,3,4$ labels the spin and orbit subspaces of $H$ in Eq. (A1). The wave function of the energy $E_{\delta}$ is given by $\Psi_{\delta, k_{x}^{\prime}}\left(y^{\prime}\right)=\sum_{s n} C_{\delta, s n}\left|\varphi_{n}\left(y^{\prime}\right)\right\rangle$. The energy dispersions of Fig. 6 are calculated numerically through the above matrix elements.

\section{Dirac semimetal slab with open boundary conditions along the $y^{\prime}$ and $z^{\prime}$ directions}

Now we consider open boundary conditions along the $y^{\prime}$ and $z^{\prime}$ directions with the thickness $L$ and the width $W$. In this case, $k_{x}^{\prime}$ is a good quantum number while $k_{y}^{\prime}$ and $k_{z}^{\prime}$ should be replaced by $k_{y}^{\prime}=-i \partial_{y^{\prime}}$ and $k_{z}^{\prime}=-i \partial_{z^{\prime}}$, respectively. The Hamiltonian $H\left(k_{x}^{\prime},-i \partial_{y^{\prime}},-i \partial_{z^{\prime}}\right)$ has the following form:

$$
H=Y_{1}+Y_{2} \partial_{y^{\prime}} \partial_{z^{\prime}}+Y_{3} \partial_{y^{\prime}}+Y_{4} \partial_{z^{\prime}}+Y_{5} \partial_{y^{\prime}}^{2}+Y_{6} \partial_{z^{\prime}}^{2},
$$

where

$$
\begin{aligned}
& Y_{1}=C_{0}+C_{2} k_{x}^{\prime 2}+\left(\begin{array}{cccc}
M_{0}+M_{2} k_{x}^{\prime 2} & A e^{i \alpha} k_{x}^{\prime} & 0 & 0 \\
A e^{-i \alpha} k_{x}^{\prime} & -M_{0}-M_{2} k_{x}^{\prime 2} & 0 & 0 \\
0 & 0 & M_{0}+M_{2} k_{x}^{\prime 2} & -A e^{-i \alpha} k_{x}^{\prime} \\
0 & 0 & -A e^{i \alpha} k_{x}^{\prime} & -M_{0}-M_{2} k_{x}^{\prime 2}
\end{array}\right), \\
& Y_{2}=\left(\begin{array}{cccc}
U_{-+-+} \sin 2 \theta & 0 & 0 & 0 \\
0 & U_{-++-} \sin 2 \theta & 0 & 0 \\
0 & 0 & U_{-+-+} \sin 2 \theta & 0 \\
0 & 0 & 0 & U_{-++-} \sin 2 \theta
\end{array}\right) \text {, } \\
& Y_{3}=\left(\begin{array}{cccc}
0 & A e^{i \alpha} \cos \theta & 0 & 0 \\
-A e^{-i \alpha} \cos \theta & 0 & 0 & 0 \\
0 & 0 & 0 & A e^{-i \alpha} \cos \theta \\
0 & 0 & -A e^{i \alpha} \cos \theta & 0
\end{array}\right) \text {, } \\
& Y_{4}=\left(\begin{array}{cccc}
0 & -A e^{i \alpha} \sin \theta & 0 & 0 \\
A e^{-i \alpha} \sin \theta & 0 & 0 & 0 \\
0 & 0 & 0 & -A e^{-i \alpha} \sin \theta \\
0 & 0 & A e^{i \alpha} \sin \theta & 0
\end{array}\right), \\
& Y_{5}=\frac{1}{2}\left(\begin{array}{cccc}
U_{----}+U_{+-+-} \cos 2 \theta & 0 & 0 & 0 \\
0 & U_{--++}+U_{+--+} \cos 2 \theta & 0 & 0 \\
0 & 0 & U_{----}+U_{+-+-} \cos 2 \theta & 0 \\
0 & 0 & 0 & U_{--++}+U_{+--+} \cos 2 \theta
\end{array}\right) \text {, }
\end{aligned}
$$



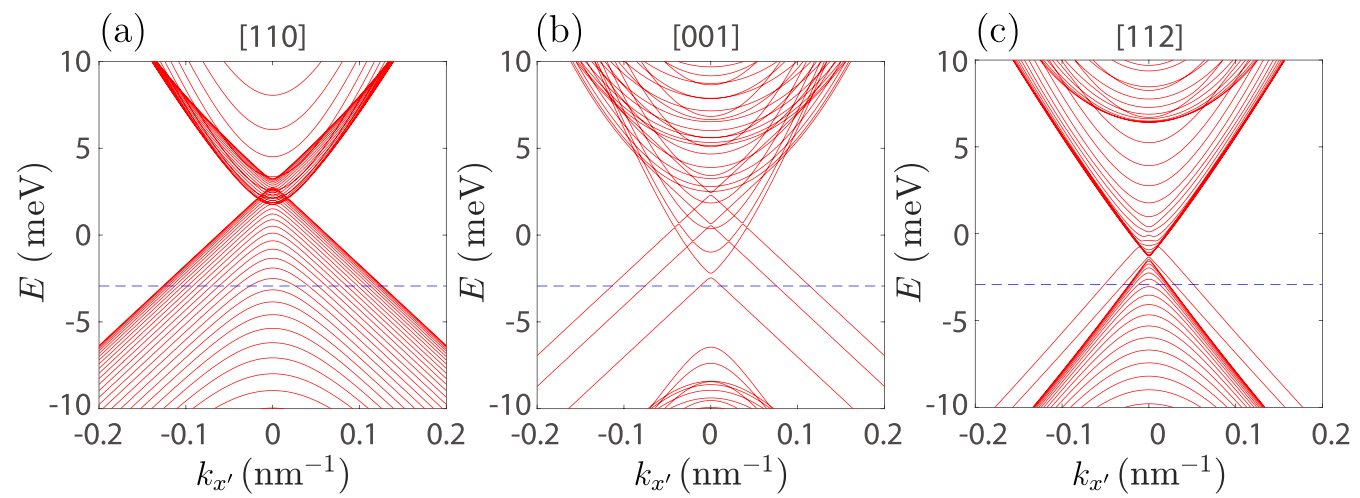

FIG. 7. Numerically calculated spectrum of the Dirac semimetal slab with open boundary conditions along $y^{\prime}$ and $z^{\prime}$ directions. The blue dashed lines label the Fermi level $E_{\mathrm{F}}=E_{\mathrm{w}}$. In (a), (b), and (c), $y^{\prime}$ correspond to [110], [001], and [112]-directions, respectively.

$$
Y_{6}=\frac{1}{2}\left(\begin{array}{cccc}
U_{----}+U_{-+-+} \cos 2 \theta & 0 & 0 & 0 \\
0 & U_{--++}+U_{-++-} \cos 2 \theta & 0 & 0 \\
0 & 0 & U_{----}+U_{-+-+} \cos 2 \theta & 0 \\
0 & 0 & 0 & U_{--++}+U_{-++-} \cos 2 \theta
\end{array}\right)
$$

with

$$
U_{ \pm \pm \pm \pm}= \pm C_{1} \pm C_{2} \pm M_{1} \pm M_{2} \text {. }
$$

For a Dirac semimetal slab with the width $W$ and the thickness $L$, we use the bases $\left|\varphi_{j}\left(y^{\prime}\right), \psi_{l}\left(z^{\prime}\right)\right\rangle=\left|\varphi_{j}\left(y^{\prime}\right)\right\rangle \otimes\left|\psi_{l}\left(z^{\prime}\right)\right\rangle$ with

$$
\begin{aligned}
& \varphi_{j}\left(y^{\prime}\right)=\sqrt{\frac{2}{L}} \sin \left[\frac{j \pi}{L}\left(y^{\prime}+\frac{L}{2}\right)\right], \quad j=1,2,3, \ldots, \\
& \psi_{l}\left(z^{\prime}\right)=\sqrt{\frac{2}{W}} \sin \left[\frac{l \pi}{W}\left(z^{\prime}+\frac{W}{2}\right)\right], \quad l=1,2,3, \ldots
\end{aligned}
$$

Then the matrix elements are given by

$$
\left\langle\varphi_{m}, \psi_{l}\left|H\left(k_{x}^{\prime},-i \partial_{y^{\prime}},-i \partial_{z^{\prime}}\right)\right| \varphi_{n}, \psi_{j}\right\rangle=\left\{\begin{array}{cl}
Y_{1}-\left(\frac{m^{2} \pi^{2} Y_{5}}{L^{2}}+\frac{j^{2} \pi^{2} Y_{6}}{W^{2}}\right) & m=n, j=l, \\
\frac{2\left[-1+(-1){ }^{j+l}\right] l j Y_{4}}{W\left(l^{2}-j^{2}\right)} & m=n, j \neq l, \\
\frac{2\left[-1+(-1)^{m+n}\right] m n Y_{3}}{L\left(n^{2}-m^{2}\right)} & m \neq n, j=l, \\
\frac{4\left[-1+(-1)^{m+n}\right]\left[-1+(-1)^{j+l}\right] m n l j Y_{2}}{L W\left(n^{2}-m^{2}\right)\left(l^{2}-j^{2}\right)} & m \neq n, j \neq l .
\end{array}\right.
$$

From the above Hamiltonian, we plot the spectrum of a Dirac semimetal slab with open boundary conditions along $y^{\prime}$ and $z^{\prime}$ directions in Fig. 7. The spectrum shows no gap for the [110] case due to the Fermi arcs. For the [001] case, the helical edge states on the side surfaces characterized by the nonzero spin Chern number can be clearly discerned in the band gap. For the [112] case, though the band gap closes, there are still helical states crossing the Fermi level.

\section{Dirac semimetal slab with open boundary conditions along the $y^{\prime}$ and $z^{\prime}$ directions under magnetic fields}

Now a uniform magnetic field $\mathbf{B}^{\prime}=\left(0, B_{y^{\prime}}, 0\right)$ is applied along the $y^{\prime}$ direction. We choose the Landau gauge $\mathbf{A}^{\prime}=\left(B_{y^{\prime}} z^{\prime}, 0,0\right)$. In the presence of the magnetic field, the wave vector should be replaced with the Peierls transformation

$$
\mathbf{k}^{\prime} \rightarrow\left(k_{x}^{\prime}-\frac{t z^{\prime}}{\ell_{y^{\prime}}^{2}},-i \partial_{y^{\prime}},-i \partial_{z^{\prime}}\right)
$$

with $\ell_{y^{\prime}}=\sqrt{\hbar /\left|e B_{y^{\prime}}\right|}$ and $t=\operatorname{sgn}\left(e B_{y^{\prime}}\right)$. In this case, the magnetic field components along the previous [100], [010], and [001] axes are given by

$$
\left[\begin{array}{l}
B_{[100]} \\
B_{[010]} \\
B_{[001]}
\end{array}\right]=\left[\begin{array}{ccc}
\cos \alpha & -\cos \theta \sin \alpha & \sin \theta \sin \alpha \\
\sin \alpha & \cos \theta \cos \alpha & -\sin \theta \cos \alpha \\
0 & \sin \theta & \cos \theta
\end{array}\right]\left[\begin{array}{c}
0 \\
B_{y^{\prime}} \\
0
\end{array}\right]
$$



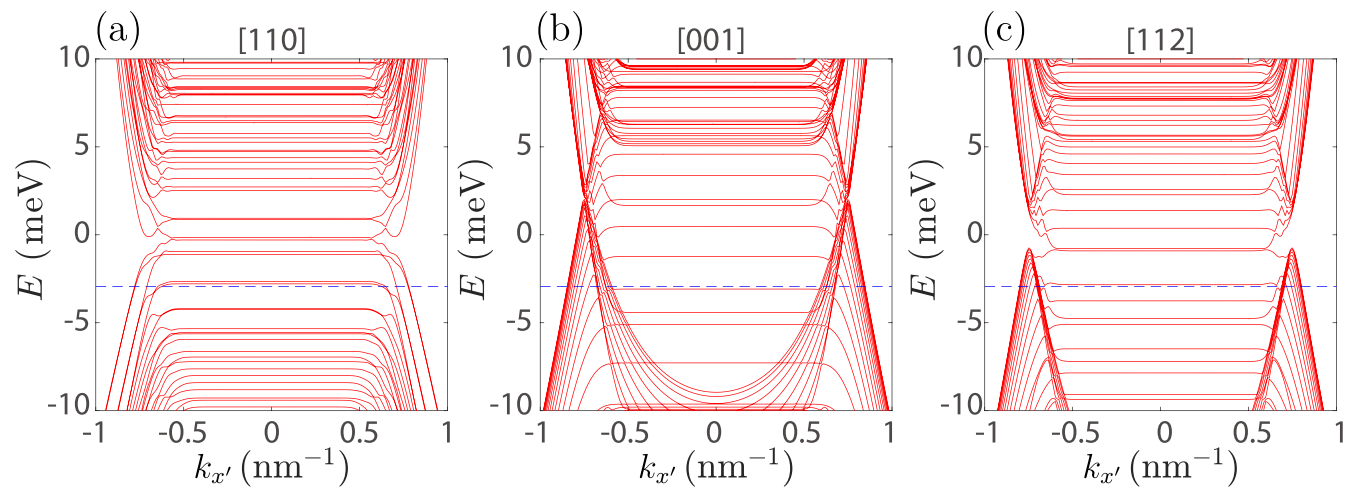

FIG. 8. Numerically calculated spectrum of the Dirac semimetal slab with open boundary conditions along the $y^{\prime}$ and $z^{\prime}$ directions with $B=$ $5 \mathrm{~T}$. The blue dashed lines label the Fermi level $E_{\mathrm{F}}=E_{\mathrm{w}}$. In (a), (b), and (c), $y^{\prime}$ correspond to [110], [001], and [112]-directions, respectively.

and the Zeeman term is

$$
\begin{aligned}
H_{\text {Zeeman }} & =\frac{\mu_{B}}{2}\left(\sigma_{x} B_{[100]}+\sigma_{y} B_{[010]}+\mid \sigma_{z} B_{[001]}\right) \otimes\left[\begin{array}{cc}
g_{s} & 0 \\
0 & g_{p}
\end{array}\right] \\
& =\frac{\mu_{B} B_{y^{\prime}}}{2}\left(\begin{array}{cccc}
g_{s} \sin \theta & 0 & -i g_{s} e^{-i \alpha} \cos \theta & 0 \\
0 & g_{p} \sin \theta & 0 & -i g_{p} e^{-i \alpha} \cos \theta \\
i g_{s} e^{i \alpha} \cos \theta & 0 & -g_{s} \sin \theta & 0 \\
0 & i g_{p} e^{i \alpha} \cos \theta & 0 & -g_{p} \sin \theta
\end{array}\right) .
\end{aligned}
$$

In the presence of the magnetic field, the Hamiltonian

$$
H_{t}=H\left(k_{x}^{\prime}-\frac{t z^{\prime}}{\ell_{y^{\prime}}^{2}},-i \partial_{y^{\prime}},-i \partial_{z^{\prime}}\right)+H_{\text {Zeeman }},
$$

has the following form:

$$
H_{t}=Y_{1}^{\prime}+Y_{2} \partial_{y^{\prime}} \partial_{z^{\prime}}+Y_{3} \partial_{y^{\prime}}+Y_{4} \partial_{z^{\prime}}+Y_{5} \partial_{y^{\prime}}^{2}+Y_{6} \partial_{z^{\prime}}^{2}+Y_{7} z^{\prime}+Y_{8} z^{\prime 2}
$$

where

$$
\begin{aligned}
& Y_{1}^{\prime}=Y_{1}+H_{\text {Zeeman }}, \\
& Y_{7}=-\frac{t}{\ell_{y^{\prime}}^{2}}\left(\begin{array}{cccc}
2\left(C_{2}+M_{2}\right) k_{x}^{\prime} & A e^{i \alpha} & 0 & 0 \\
A e^{-i \alpha} & 2\left(C_{2}-M_{2}\right) k_{x}^{\prime} & 0 & 0 \\
0 & 0 & 2\left(C_{2}+M_{2}\right) k_{x}^{\prime} & -A e^{-i \alpha} \\
0 & 0 & -A e^{i \alpha} & 2\left(C_{2}-M_{2}\right) k_{x}^{\prime}
\end{array}\right) \text {, } \\
& Y_{8}=\frac{1}{\ell_{y^{\prime}}^{4}}\left(\begin{array}{cccc}
C_{2}+M_{2} & 0 & 0 & 0 \\
0 & C_{2}-M_{2} & 0 & 0 \\
0 & 0 & C_{2}+M_{2} & 0 \\
0 & 0 & 0 & C_{2}-M_{2}
\end{array}\right) .
\end{aligned}
$$

In the basis of $\left|\varphi_{j}(y), \psi_{l}(z)\right\rangle=\left|\varphi_{j}(y)\right\rangle \otimes\left|\psi_{l}(z)\right\rangle[$ [Eq. (A19)], the matrix elements are given by

$$
\begin{aligned}
& \left\langle\varphi_{m}, \psi_{l}\left|H_{t}\left(k_{x}^{\prime},-i \partial_{y^{\prime}},-i \partial_{z^{\prime}}\right)\right| \varphi_{n}, \psi_{j}\right\rangle \\
& =\left\{\begin{array}{cl}
Y_{1}^{\prime}-\left(\frac{m^{2} \pi^{2} Y_{5}}{L^{2}}+\frac{j^{2} \pi^{2} Y_{6}}{W^{2}}\right)+\frac{W^{2}}{12}\left(1-\frac{6}{j^{2} \pi^{2}}\right) Y_{8} & m=n, j=l, \\
\frac{2 l j\left\{\left[-1+(-1)^{j+l}\right]\left(i^{2}-j^{2}\right) \pi^{2} Y_{4}+2\left[-1+(-1)^{j+l}\right] W^{2} Y_{7}+2\left[1+(-1)^{j+l}\right] W^{3} Y_{8}\right\}}{W\left(l^{2}-j^{2}\right)^{2} \pi^{2}} & m=n, j \neq l, \\
\frac{2\left[-1+(-1)^{m+n}\right] m n Y_{3}}{L\left(n^{2}-m^{2}\right)} & m \neq n, j=l, \\
\frac{4\left[-1+(-1)^{m+n}\left[-1+(-1) j^{j+l}\right] m n l j Y_{2}\right.}{L W\left(n^{2}-m^{2}\right)\left(l^{2}-j^{2}\right)} & m \neq n, j \neq l .
\end{array}\right.
\end{aligned}
$$

From the above Hamiltonian, we plot the spectrum of a Dirac semimetal slab with open boundary conditions along the $y^{\prime}$ and $z^{\prime}$ directions in Fig. 8. For the slab along [001] or [112] direction, the helical edge states are robust against the magnetic field, and contribute to the quantized Hall conductivity. 


\section{APPENDIX B: SPIN CHERN NUMBER}

\section{Spin Chern number for the slab grown along the [001] direction}

For the slab grown along the [001] direction $(\alpha=-\pi / 4, \theta=\pi / 2)$, it is found $X_{2}=0$. The Hamiltonian of the Dirac semimetal slab from Eq. (A10) has the form

$$
\mathcal{H}=\left(\begin{array}{ccccc}
\mathcal{H}_{11} & 0 & 0 & 0 & \ldots \\
0 & \mathcal{H}_{22} & 0 & 0 & \ldots \\
0 & 0 & \mathcal{H}_{33} & 0 & \ldots \\
0 & 0 & 0 & \mathcal{H}_{44} & \ldots \\
\vdots & \vdots & \vdots & \vdots & \ddots
\end{array}\right),
$$

which can be regarded as a superposition of different blocks. Each block is equivalent to a Bernevig-Hughes-Zhang (BHZ) model Hamiltonian describing a 2D topological insulator, whose topological invariant can be defined by using the spin Chern number as

$$
C_{\mathrm{eff}}^{s}(n)=\frac{C_{\mathrm{eff}}^{\uparrow}(n)-C_{\mathrm{eff}}^{\downarrow}(n)}{2},
$$

where

$$
C_{\mathrm{eff}}^{\uparrow, \downarrow}(n)= \pm \frac{\operatorname{sgn}\left(M_{0}+M_{1} n^{2} \pi^{2} / L^{2}\right)-\operatorname{sgn}\left(M_{2}\right)}{2},
$$

are the valence-band Chern numbers of the spin-up and spin-down blocks of the $n$th block.

\section{Spin Chern number for the slab grown along the [112] direction}

For the slab is along the [112] direction $(\alpha=-\pi / 4, \theta=\arctan \sqrt{2})$, each subband of the Hamiltonian obtained through the quantum well approximation couple together because $X_{2} \neq 0$. Therefore, we adopt the tight-binding approximation to discretize the Hamiltonian $H\left(k_{x}^{\prime}, k_{y}^{\prime}, k_{z}^{\prime}\right)$ into a quasi-two-dimensional model having the form

$$
H^{\mathrm{tb}}\left(k_{x}^{\prime}, k_{y}^{\prime}\right)=\sum_{i} h_{0} c_{i}^{\dagger} c_{i}+T_{y} c_{i}^{\dagger} c_{i+1}+T_{y}^{\dagger} c_{i}^{\dagger} c_{i-1},
$$

where

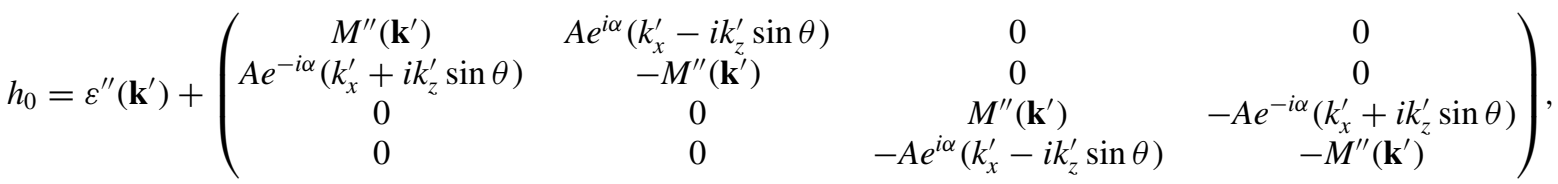

$$
\begin{aligned}
& T_{y}=\frac{1}{2}\left(\begin{array}{cccc}
U_{---}+U_{+-+-} R & A e^{i \alpha} \cos \theta & 0 & 0 \\
-A e^{-i \alpha} \cos \theta & U_{--++}+U_{+--+} R & 0 & 0 \\
0 & 0 & U_{----}+U_{+-+-} R & A e^{-i \alpha} \cos \theta \\
0 & 0 & -A e^{i \alpha} \cos \theta & U_{--++}+U_{+--+} R
\end{array}\right) \text {, }
\end{aligned}
$$

with

$$
\begin{gathered}
\varepsilon^{\prime \prime}\left(\mathbf{k}^{\prime}\right)=C_{0}+C_{1}\left(2 \sin ^{2} \theta+k_{z}^{\prime 2} \cos ^{2} \theta\right)+C_{2}\left(2 \cos ^{2} \theta+k_{x}^{\prime 2}+k_{z}^{\prime 2} \sin ^{2} \theta\right), \\
M^{\prime \prime}\left(\mathbf{k}^{\prime}\right)=M_{0}+M_{1}\left(2 \sin ^{2} \theta+k_{z}^{\prime 2} \cos ^{2} \theta\right)+M_{2}\left(2 \cos ^{2} \theta+k_{x}^{\prime 2}+k_{z}^{\prime 2} \sin ^{2} \theta\right), \\
R=\cos 2 \theta-i k_{z}^{\prime} \sin 2 \theta .
\end{gathered}
$$

From the above Hamiltonian, one can calculate the spin Chern number of the occupied valence bands.

\section{APPENDIX C: HALL CONDUCTANCE OF A DIRAC SEMIMETAL SLAB UNDER MAGNETIC FIELD}

By introducing the ladder operators

$$
\begin{array}{r}
a=\frac{\ell_{y^{\prime}}}{\sqrt{2}}\left[k_{x}^{\prime}-\frac{t_{y^{\prime}} z^{\prime}}{\ell_{y^{\prime}}^{2}}-t_{y} i\left(-i \partial_{z^{\prime}}\right)\right], \\
a^{\dagger}=\frac{\ell_{y^{\prime}}}{\sqrt{2}}\left[k_{x}^{\prime}-\frac{t_{y^{\prime}} z^{\prime}}{\ell_{y^{\prime}}^{2}}+t_{y} i\left(-i \partial_{z^{\prime}}\right)\right] .
\end{array}
$$


The Hamiltonian $H_{t}$ in Eq. (A25) can be written as

$$
H_{t}=T_{1}+T_{2} \partial_{y^{\prime}}+T_{3} \partial_{y^{\prime}}^{2}
$$

where

$$
\begin{gathered}
T_{1}=T_{1}^{0}+T_{1}^{a} a+T_{1}^{a^{\dagger}} a^{\dagger}+T_{1}^{a a^{\dagger}} a a^{\dagger}+T_{1}^{a^{\dagger} a} a^{\dagger} a+T_{1}^{a^{2}} a^{2}+T_{1}^{a^{\dagger 2}} a^{\dagger 2}, \\
T_{2}=T_{2}^{0}+T_{2}^{a} a+T_{2}^{a^{\dagger}} a^{\dagger}, \\
T_{3}=T_{3}^{0},
\end{gathered}
$$

with

$$
\begin{aligned}
& T_{1}^{0}=C_{0}+\left(\begin{array}{cccc}
M_{0} & 0 & 0 & 0 \\
0 & -M_{0} & 0 & 0 \\
0 & 0 & M_{0} & 0 \\
0 & 0 & 0 & -M_{0}
\end{array}\right)+H_{\text {Zeeman }} \\
& T_{1}^{a}=\frac{1}{\sqrt{2} \ell_{y^{\prime}} t_{y^{\prime}}}\left(\begin{array}{cccc}
0 & A e^{i \alpha}\left(t_{y}+\sin \theta\right) & 0 & 0 \\
A e^{-i \alpha}\left(t_{y}-\sin \theta\right) & 0 & 0 & 0 \\
0 & 0 & 0 & A e^{-i \alpha}\left(-t_{y}+\sin \theta\right) \\
0 & 0 & -A e^{i \alpha}\left(t_{y}+\sin \theta\right) & 0
\end{array}\right), \\
& T_{1}^{a^{\dagger}}=\frac{1}{\sqrt{2} \ell_{y^{\prime}} t_{y^{\prime}}}\left(\begin{array}{cccc}
0 & A e^{i \alpha}\left(t_{y}-\sin \theta\right) & 0 & 0 \\
A e^{-i \alpha}\left(t_{y}+\sin \theta\right) & 0 & 0 & 0 \\
0 & 0 & 0 & -A e^{-i \alpha}\left(t_{y}+\sin \theta\right) \\
0 & 0 & -A e^{i \alpha}\left(t_{y}-\sin \theta\right) & 0
\end{array}\right) \text {, } \\
& T_{1}^{a a^{\dagger}}=\frac{1}{4 \ell_{y^{\prime}}^{2}}\left(\begin{array}{cccc}
U_{++++}^{\prime}+U_{+-+-} \cos 2 \theta & 0 & 0 & 0 \\
0 & U_{++--}^{\prime}+U_{+--+} \cos 2 \theta & 0 & 0 \\
0 & 0 & U_{++++}^{\prime}+U_{+-+-} \cos 2 \theta & 0 \\
0 & 0 & 0 & U_{++--}^{\prime}+U_{+--+} \cos 2 \theta
\end{array}\right) \text {, } \\
& T_{1}^{a^{\dagger} a}=\frac{1}{4 \ell_{y^{\prime}}^{2}}\left(\begin{array}{cccc}
U_{++++}^{\prime}+U_{+-+-} \cos 2 \theta & 0 & 0 & 0 \\
0 & U_{++--}^{\prime}+U_{+-++} \cos 2 \theta & 0 & 0 \\
0 & 0 & U_{++++}^{\prime}+U_{+-+-} \cos 2 \theta & 0 \\
0 & 0 & 0 & U_{++--}^{\prime}+U_{+--+} \cos 2 \theta
\end{array}\right) \\
& T_{1}^{a^{2}}=T_{1}^{a^{\dagger 2}}=\left(\begin{array}{cccc}
2 U_{-+-+} \cos ^{2} \theta & 0 & 0 & 0 \\
0 & 2 U_{-++-} \cos ^{2} \theta & 0 & 0 \\
0 & 0 & 2 U_{-+-+} \cos ^{2} \theta & 0 \\
0 & 0 & 0 & 2 U_{-++-} \cos ^{2} \theta
\end{array}\right) \\
& T_{2}^{0}=\left(\begin{array}{cccc}
0 & A e^{i \alpha} \cos \theta & 0 & 0 \\
-A e^{-i \alpha} \cos \theta & 0 & 0 & 0 \\
0 & 0 & 0 & A e^{-i \alpha} \cos \theta \\
0 & 0 & -A e^{i \alpha} \cos \theta & 0
\end{array}\right) \\
& T_{2}^{a}=\frac{1}{\sqrt{2} \ell_{y^{\prime}} t_{y^{\prime}}}\left(\begin{array}{cccc}
U_{+-+-} \sin 2 \theta & 0 & 0 & 0 \\
0 & U_{+--+} \sin 2 \theta & 0 & 0 \\
0 & 0 & U_{+-+-} \sin 2 \theta & 0 \\
0 & 0 & 0 & U_{+--+} \sin 2 \theta
\end{array}\right) \text {, } \\
& T_{2}^{a^{\dagger}}=\frac{1}{\sqrt{2} \ell_{y^{\prime}} t_{y^{\prime}}}\left(\begin{array}{cccc}
U_{-+-+} \sin 2 \theta & 0 & 0 & 0 \\
0 & U_{-++-} \sin 2 \theta & 0 & 0 \\
0 & 0 & U_{-+-+} \sin 2 \theta & 0 \\
0 & 0 & 0 & U_{-++-} \sin 2 \theta
\end{array}\right) \text {, } \\
& T_{3}^{0}=\frac{1}{2}\left(\begin{array}{cccc}
U_{----}+U_{+-+-} \cos 2 \theta & 0 & 0 & 0 \\
0 & U_{--++}+U_{+--+} \cos 2 \theta & 0 & 0 \\
0 & 0 & U_{----}+U_{+-+-\cos 2 \theta} & 0 \\
0 & 0 & 0 & U_{--++}+U_{+--+} \cos 2 \theta
\end{array}\right),
\end{aligned}
$$


and $U_{ \pm \pm \pm \pm}^{\prime}= \pm C_{1} \pm 3 C_{2} \pm M_{1} \pm 3 M_{2}$. In the basis of $\left|\varphi_{n}, v\right\rangle=\left|\varphi_{n}\left(y^{\prime}\right)\right\rangle \otimes|v\rangle$, where $|v\rangle$ is the harmonic oscillator eigenfunction given by

$$
\langle z \mid v\rangle=\phi_{\nu}(z)=\frac{1}{\sqrt{\pi^{1 / 2} 2^{v} v ! \ell_{y^{\prime}}}} \exp \left[-\frac{\left(z-z_{0}\right)^{2}}{2 \ell_{y^{\prime}}^{2}}\right] \mathcal{H}_{\nu}\left(\frac{z-z_{0}}{\ell_{y^{\prime}}}\right),
$$

and $\mathcal{H}_{v}$ is the Hermite polynomial and the guiding center $z_{0}=t k_{x} \ell_{y^{\prime}}^{2}$. With the basis, the matrix elements of the Hamiltonian in the magnetic field are found as

$$
H_{t}^{m n, v^{\prime} v}=\left\langle v^{\prime}, \varphi_{m}\left|H_{t}\right| \varphi_{n}, v\right\rangle= \begin{cases}\left\langle v^{\prime}\left|T_{1}\right| v\right\rangle-\frac{n^{2} \pi^{2}}{L^{2}}\left\langle v^{\prime}\left|T_{3}\right| v\right\rangle, & m=n, \\ -\frac{2\left[-1+(-1)^{n+m}\right] n m}{L\left(n^{2}-m^{2}\right)}\left\langle v^{\prime}\left|T_{2}\right| v\right\rangle, & m \neq n .\end{cases}
$$

Because

$$
\begin{gathered}
a|v\rangle=\sqrt{v}|v-1\rangle, \\
a^{\dagger}|v\rangle=\sqrt{v+1}|v+1\rangle,
\end{gathered}
$$

then $\left\langle v^{\prime}\left|T_{1,2,3}\right| v\right\rangle$ can be obtained through the following relationships:

$$
\begin{aligned}
& \left\langle v^{\prime}|a| v\right\rangle \rightarrow\left(\begin{array}{cccccc}
0 & 0 & 0 & 0 & 0 & \ddots \\
1 & 0 & 0 & 0 & 0 & \ddots \\
0 & \sqrt{2} & 0 & 0 & 0 & \ddots \\
0 & 0 & \sqrt{3} & 0 & 0 & \ddots \\
0 & 0 & 0 & \sqrt{4} & 0 & \ddots \\
\ddots & \ddots & \ddots & \ddots & \ddots & \ddots
\end{array}\right), \quad\left\langle v^{\prime}\left|a^{\dagger}\right| v\right\rangle \rightarrow\left(\begin{array}{cccccc}
0 & 1 & 0 & 0 & 0 & \ddots \\
0 & 0 & \sqrt{2} & 0 & 0 & \ddots \\
0 & 0 & 0 & \sqrt{3} & 0 & \ddots \\
0 & 0 & 0 & 0 & \sqrt{4} & \ddots \\
0 & 0 & 0 & 0 & 0 & \ddots \\
\ddots & \ddots & \ddots & \ddots & \ddots & \ddots
\end{array}\right) \\
& \left\langle v^{\prime}\left|a a^{\dagger}\right| v\right\rangle \rightarrow\left(\begin{array}{cccccc}
1 & 0 & 0 & 0 & 0 & \ddots \\
0 & 2 & 0 & 0 & 0 & \ddots \\
0 & 0 & 3 & 0 & 0 & \ddots \\
0 & 0 & 0 & 4 & 0 & \ddots \\
0 & 0 & 0 & 0 & 5 & \ddots \\
\ddots & \ddots & \ddots & \ddots & \ddots & \ddots
\end{array}\right), \quad\left\langle v^{\prime}\left|a^{\dagger} a\right| v\right\rangle \rightarrow\left(\begin{array}{cccccc}
0 & 0 & 0 & 0 & 0 & \ddots \\
0 & 1 & 0 & 0 & 0 & \ddots \\
0 & 0 & 2 & 0 & 0 & \ddots \\
0 & 0 & 0 & 3 & 0 & \ddots \\
0 & 0 & 0 & 0 & 4 & \ddots \\
\ddots & \ddots & \ddots & \ddots & \ddots & \ddots
\end{array}\right), \\
& \left\langle v^{\prime}\left|a^{2}\right| \nu\right\rangle \rightarrow\left(\begin{array}{cccccc}
0 & 0 & 0 & 0 & 0 & \ddots \\
0 & 0 & 0 & 0 & 0 & \ddots \\
\sqrt{2} & 0 & 0 & 0 & 0 & \ddots \\
0 & \sqrt{6} & 0 & 0 & 0 & \ddots \\
0 & 0 & \sqrt{12} & 0 & 0 & \ddots \\
\ddots & \ddots & \ddots & \ddots & \ddots & \ddots
\end{array}\right), \quad\left\langle v^{\prime}\left|a^{\dagger 2}\right| v\right\rangle \rightarrow\left(\begin{array}{cccccc}
0 & 0 & \sqrt{2} & 0 & 0 & \ddots \\
0 & 0 & 0 & \sqrt{6} & 0 & \ddots \\
0 & 0 & 0 & 0 & \sqrt{12} & \ddots \\
0 & 0 & 0 & 0 & 0 & \ddots \\
0 & 0 & 0 & 0 & 0 & \ddots \\
\ddots & \ddots & \ddots & \ddots & \ddots & \ddots
\end{array}\right)
\end{aligned}
$$

From the above Hamiltonian, we can obtain the energy of the Landau level $E_{\delta}$, and the wave function is given by

$$
\left|\Psi_{\delta}\right\rangle=\sum_{s n v} C_{\delta, s n v}|n, v\rangle,
$$

Here $s=1,2,3,4$ describes the spin and orbit subspace.

Now we calculate the Hall conductivity defined by $\sigma_{\mathrm{H}}=j_{x^{\prime}} / E_{z^{\prime}}$, where the current $j_{x^{\prime}}$ is applied along the $x^{\prime}$ direction and the induced electric field $E_{z^{\prime}}$ is along the $z^{\prime}$ direction. The velocity operators given by

$$
\begin{aligned}
& v_{x}^{m n}=\frac{1}{\hbar}\left\langle m\left|v_{x}\right| n\right\rangle=\frac{1}{\hbar} \partial \mathcal{H}_{m n} / \partial k_{x}^{\prime} \\
& =\frac{1}{\hbar}\left\{\begin{array}{cccc}
2 k_{x}^{\prime}\left(C_{2}+M_{2}\right) & A e^{i \alpha} & 0 & 0 \\
A e^{-i \alpha} & 2 k_{x}^{\prime}\left(C_{2}-M_{2}\right) & 0 & 0 \\
0 & 0 & 2 k_{x}^{\prime}\left(C_{2}+M_{2}\right) & -A e^{-i \alpha} \\
0 & 0 & -A e^{i \alpha} & 2 k_{x}^{\prime}\left(C_{2}-M_{2}\right)
\end{array}\right), \quad m=n,
\end{aligned}
$$




$$
\begin{aligned}
v_{z}^{m n} & =\frac{1}{\hbar}\left\langle m\left|v_{z}\right| n\right\rangle=\frac{1}{\hbar} \partial \mathcal{H}_{m n} / \partial k_{z}^{\prime} \\
& =\frac{1}{\hbar}\left\{\begin{array}{cccc}
K_{1} k_{z}^{\prime} & -i A e^{i \alpha} \sin \theta & 0 & 0 \\
i A e^{-i \alpha} \sin \theta & K_{2} k_{z}^{\prime} & 0 & 0 \\
0 & 0 & K_{1} k_{z}^{\prime} & -i A e^{-i \alpha} \sin \theta \\
0 & 0 & i A e^{i \alpha} \sin \theta & K_{2} k_{z}^{\prime}
\end{array}\right), \quad m=n, \\
\frac{2 i\left[-1+(-1)^{m+n}\right] m n \sin 2 \theta}{L\left(m^{2}-n^{2}\right)}\left(\begin{array}{cccc}
U_{+-+-} & 0 & 0 & 0 \\
0 & U_{+--+} & 0 & 0 \\
0 & 0 & U_{+-+-} & 0 \\
0 & 0 & 0 & U_{+--+}
\end{array}\right), m \neq n, & m,
\end{aligned}
$$

with $K_{1}=\left(U_{++++}+U_{+-+-} \cos 2 \theta\right)$ and $K_{2}=\left(U_{++--}+U_{+--+} \cos 2 \theta\right)$. When the magnetic field is introduced, the velocity operator becomes $v_{x, z}\left(k_{x}^{\prime}, k_{z}^{\prime}\right) \rightarrow v_{x, z}\left(k_{x}^{\prime}-\frac{t z^{\prime}}{\ell_{y^{\prime}}^{2}},-i \partial_{z^{\prime}}\right)$. The matrix elements of the velocity operators are

$$
\begin{aligned}
& \left\langle\Psi_{\delta}\left|v_{x}\right| \Psi_{\delta^{\prime}}\right\rangle=\frac{1}{\hbar} \sum_{m n v v^{\prime}}\left\langle v^{\prime}\left|C_{\delta, m v^{\prime}}^{\dagger} v_{x}^{m n} C_{\delta^{\prime}, n v}\right| v\right\rangle, \\
& \left\langle\Psi_{\delta^{\prime}}\left|v_{z}\right| \Psi_{\delta}\right\rangle=\frac{1}{\hbar} \sum_{m n v v^{\prime}}\left\langle v^{\prime}\left|C_{\delta^{\prime}, m v^{\prime}}^{\dagger} v_{z}^{m n} C_{\delta, n v}\right| v\right\rangle,
\end{aligned}
$$

where $v_{x}^{m n}$ is a $4 \times 4$ matrix given in Eqs. (C26) and (C28) and

$$
C_{\delta^{\prime}, n v}=\left(\begin{array}{l}
C_{\delta^{\prime}, n v}^{1} \\
C_{\delta^{\prime}, n v}^{2} \\
C_{\delta^{\prime}, n v}^{3} \\
C_{\delta^{\prime}, n v}^{4}
\end{array}\right),
$$

is a $4 \times 1$ vector. With the help of ladder operators, we have

$$
\begin{aligned}
& C_{\delta, m v}^{\dagger} v_{x}^{m n} C_{\delta^{\prime}, n v}=S_{x}^{\delta \delta^{\prime} m n v^{\prime} v}+S_{x, a}^{\delta \delta^{\prime} m n v^{\prime} v} a+S_{x, a^{\dagger}}^{\delta \delta^{\prime} m n v^{\prime} v} a^{\dagger}, \\
& C_{\delta^{\prime}, m v}^{\dagger} v_{z}^{m n} C_{\delta, n v}=S_{z}^{\delta^{\prime} \delta m n v^{\prime} v}+S_{z, a}^{\delta^{\prime} \delta m n v^{\prime} v} a+S_{z, a^{\dagger}}^{\delta^{\prime} \delta m v^{\prime} v} a^{\dagger} .
\end{aligned}
$$

Then

$$
\begin{aligned}
\left\langle\Psi_{\delta}\left|v_{x}\right| \Psi_{\delta^{\prime}}\right\rangle & =\frac{1}{\hbar} \sum_{m n v v^{\prime}} S_{x}^{\delta \delta^{\prime} m n v^{\prime} v} \delta_{v^{\prime} v}+S_{x, a}^{\delta \delta^{\prime} m n v^{\prime} v} \sqrt{v} \delta_{v^{\prime} v-1}+S_{x, a^{\dagger}}^{\delta \delta^{\prime} m n v^{\prime} v} \sqrt{v+1} \delta_{v^{\prime} v+1}, \\
\left\langle\Psi_{\delta}\left|v_{z}\right| \Psi_{\delta^{\prime}}\right\rangle & =\frac{1}{\hbar} \sum_{m n v v^{\prime}} S_{z}^{\delta \delta^{\prime} m n v^{\prime}} \delta_{v^{\prime} v}+S_{z, a}^{\delta \delta^{\prime} m n v^{\prime}} \sqrt{v} \delta_{v^{\prime} v-1}+S_{z, a^{\dagger}}^{\delta \delta^{\prime} m n v^{\prime}} \sqrt{v+1} \delta_{v^{\prime} v+1} .
\end{aligned}
$$

Take the velocities in Eqs. (C26) and (C28) into Eqs. (C29) and (C30), we have

$$
\begin{aligned}
& v_{x}^{\delta \delta^{\prime}}=\sum_{m n v v^{\prime}} S_{x}^{\delta \delta^{\prime} m n v^{\prime} v} \delta_{\nu^{\prime} v}=\delta_{m n} \delta_{\nu^{\prime} \nu} A\left[e^{-i \alpha}\left(C_{\delta, m v^{\prime}}^{2 *} C_{\delta^{\prime}, n v}^{1}-C_{\delta, m v^{\prime}}^{3 *} C_{\delta^{\prime}, n v}^{4}\right)+e^{i \alpha}\left(C_{\delta, m v^{\prime}}^{1 *} C_{\delta^{\prime}, n v}^{2}-C_{\delta, m v^{\prime}}^{4 *} C_{\delta^{\prime}, n v}^{3}\right)\right], \\
& v_{x, a}^{\delta \delta^{\prime}}=\sum_{m n v v^{\prime}} S_{x, a}^{\delta \delta^{\prime} m n v^{\prime} v} \sqrt{v} \delta_{v^{\prime} v-1} \\
& =\delta_{m n} \delta_{\nu^{\prime} v-1} \frac{\sqrt{2 v}}{\ell_{y^{\prime}}}\left[\left(C_{2}+M_{2}\right)\left(C_{\delta, m v^{\prime}}^{1 *} C_{\delta^{\prime}, n v}^{1}+C_{\delta, m \nu^{\prime}}^{3 *} C_{\delta^{\prime}, n v}^{3}\right)+\left(C_{2}-M_{2}\right)\left(C_{\delta, m v^{\prime}}^{2 *} C_{\delta^{\prime}, n v}^{2}+C_{\delta, m v^{\prime}}^{4 *} C_{\delta^{\prime}, n v}^{4}\right)\right], \\
& v_{x, a^{\dagger}}^{\delta \delta^{\prime}}=\sum_{m n v v^{\prime}} S_{x, a^{\dagger}}^{\delta \delta^{\prime} m n v^{\prime} v} \sqrt{v+1} \delta_{v^{\prime} v+1} \\
& =\delta_{m n} \delta_{\nu^{\prime} v+1} \frac{\sqrt{2(v+1)}}{\ell_{y^{\prime}}}\left[\left(C_{2}+M_{2}\right)\left(C_{\delta, m \nu^{\prime}}^{1 *} C_{\delta^{\prime}, n v}^{1}+C_{\delta, m \nu^{\prime}}^{3 *} C_{\delta^{\prime}, n v}^{3}\right)+\left(C_{2}-M_{2}\right)\left(C_{\delta, m \nu^{\prime}}^{2 *} C_{\delta^{\prime}, n v}^{2}+C_{\delta, m \nu^{\prime}}^{4 *} C_{\delta^{\prime}, n v}^{4}\right)\right] \text {, } \\
& v_{z}^{\delta \delta^{\prime}}=\sum_{m n v v^{\prime}} S_{z}^{\delta \delta^{\prime} m n v^{\prime}} \delta_{v^{\prime} v}=\delta_{m n} \delta_{v^{\prime} v} i A\left[e^{-i \alpha}\left(C_{\delta, m v^{\prime}}^{2 *} C_{\delta^{\prime}, n v}^{1}-C_{\delta, m v^{\prime}}^{3 *} C_{\delta^{\prime}, n v}^{4}\right)+e^{i \alpha}\left(C_{\delta, m v^{\prime}}^{4 *} C_{\delta^{\prime}, n v}^{3}-C_{\delta, m v^{\prime}}^{1 *} C_{\delta^{\prime}, n v}^{2}\right)\right] \\
& +\sum_{m \neq n} \delta_{\nu^{\prime} v} \frac{2 i\left[-1+(-1)^{m+n}\right] m n \sin 2 \theta}{L\left(m^{2}-n^{2}\right)}\left[U_{+-+-} C_{\delta, m v^{\prime}}^{1 *} C_{\delta^{\prime}, n v}^{1}+U_{+--+} C_{\delta, m v^{\prime}}^{2 *} C_{\delta^{\prime}, n v}^{2}\right. \\
& \left.+U_{+-+-} C_{\delta, m v^{\prime}}^{3 *} C_{\delta^{\prime}, n v}^{3}+U_{+--+} C_{\delta, m v^{\prime}}^{4 *} C_{\delta^{\prime}, n v}^{4}\right]
\end{aligned}
$$




$$
\begin{aligned}
& v_{z, a}^{\delta \delta^{\prime}}=\sum_{m n v v^{\prime}} S_{x, a}^{\delta \delta^{\prime} m n v^{\prime} v} \sqrt{v} \delta_{\nu^{\prime} v-1} \\
& =\delta_{m n} \delta_{\nu^{\prime} \nu-1} \frac{i \sqrt{v}}{\sqrt{2} \ell_{y^{\prime}} t_{y^{\prime}}}\left[\left(U_{++++}+U_{+-+-} \cos 2 \theta\right) C_{\delta, m \nu^{\prime}}^{1 *} C_{\delta^{\prime}, n v}^{1}+\left(U_{++--}+U_{+--+} \cos 2 \theta\right) C_{\delta, m \nu^{\prime}}^{2 *} C_{\delta^{\prime}, n v}^{2}\right. \\
& \left.+\left(U_{++++}+U_{+-+-} \cos 2 \theta\right) C_{\delta, m v^{\prime}}^{3 *} C_{\delta^{\prime}, n v}^{3}+\left(U_{++--}+U_{+--+} \cos 2 \theta\right) C_{\delta, m \nu^{\prime}}^{4 *} C_{\delta^{\prime}, n v}^{4}\right), \\
& v_{z, a^{\dagger}}^{\delta \delta^{\prime}}=\sum_{m n v v^{\prime}} S_{z, a^{\dagger}}^{\delta \delta^{\prime} m n v^{\prime}} \sqrt{v+1} \delta_{v^{\prime} v+1} \\
& =-\delta_{m n} \delta_{\nu^{\prime} v+1} \frac{i \sqrt{v+1}}{\sqrt{2} \ell_{y^{\prime}} t_{y^{\prime}}}\left[\left(C_{2}+M_{2}\right)\left(C_{\delta, m v^{\prime}}^{1 *} C_{\delta^{\prime}, n v}^{1}+C_{\delta, m v^{\prime}}^{3 *} C_{\delta^{\prime}, n v}^{3}\right)+\left(C_{2}-M_{2}\right)\left(C_{\delta, m \nu^{\prime}}^{2 *} C_{\delta^{\prime}, n v}^{2}+C_{\delta, m \nu^{\prime}}^{4 *} C_{\delta^{\prime}, n v}^{4}\right)\right] \\
& \left.+\left(U_{++++}+U_{+-+-} \cos 2 \theta\right) C_{\delta, m \nu^{\prime}}^{3 *} C_{\delta^{\prime}, n \nu}^{3}+\left(U_{++--}+U_{+--+} \cos 2 \theta\right) C_{\delta, m \nu^{\prime}}^{4 *} C_{\delta^{\prime}, n \nu}^{4}\right) .
\end{aligned}
$$

Further we can define $\bar{v}_{x}^{\delta \delta^{\prime}}=\frac{\hbar \ell_{y^{\prime}}}{\sqrt{2}}\left\langle\Psi_{\delta}\left|v_{x}\right| \Psi_{\delta^{\prime}}\right\rangle$ and $\bar{v}_{z}^{\delta \delta^{\prime}}=\frac{\hbar \ell_{y^{\prime}}}{i t_{y^{\prime}} \sqrt{2}}\left\langle\Psi_{\delta}\left|v_{z}\right| \Psi_{\delta^{\prime}}\right\rangle$, which satisfy $\bar{v}_{x}^{\delta^{\prime} \delta}=\left(\bar{v}_{x}^{\delta \delta^{\prime}}\right)^{*}$ and $\bar{v}_{z}^{\delta \delta^{\prime}}=-\left(\bar{v}_{z}^{\delta^{\prime} \delta}\right)^{*}$. Using the Kubo formula, the Hall conductivity at zero temperature reduces to

$$
\sigma_{\mathrm{H}}=t \frac{e^{2}}{h} \frac{4}{L \ell_{B}^{4}} \sum_{\substack{E_{\delta}<E_{F} \\ E_{\delta^{\prime}}>E_{F}}} \frac{\operatorname{Re}\left(\bar{v}_{x}^{\delta \delta^{\prime}} \bar{v}_{z}^{\delta^{\prime} \delta}\right)}{\left(E_{\delta}-E_{\delta^{\prime}}\right)^{2}+\Gamma^{2}} .
$$

Then, we can define $2 \mathrm{D}$ sheet conductivity $\sigma_{\mathrm{H}}^{s}=\sigma_{\mathrm{H}} L$.

Moreover, in numerical calculations, the matrix operations are much faster than the for loop. Therefore, in the following contents, we describe the numerical method calculating the conductance. For example, we use a model with two states below the chemical potential, and three states above the chemical potential. We limit the basis $n=1,2,3$ and $v=0,1,2$. Then, we can define the eight matrices

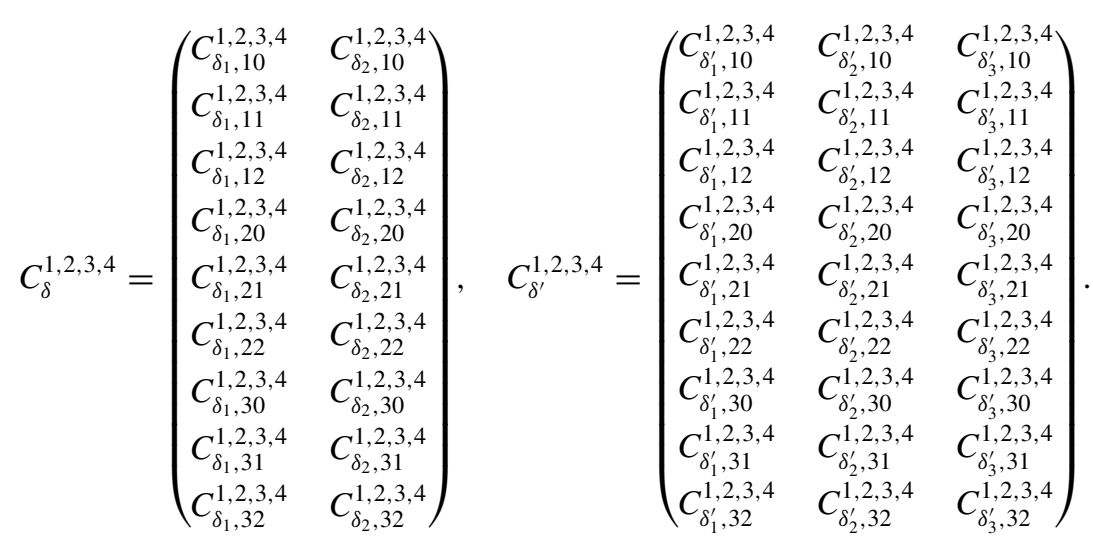

The velocity matrices can be obtained by

$$
\begin{aligned}
& {\left[v_{x}\right]=\left(\begin{array}{ccc}
v_{x}^{\delta_{1} \delta_{1}^{\prime}} & v_{x}^{\delta_{1} \delta_{2}^{\prime}} & v_{x}^{\delta_{1} \delta_{3}^{\prime}} \\
v_{x}^{\delta_{2} \delta_{1}^{\prime}} & v_{x}^{\delta_{2} \delta_{2}^{\prime}} & v_{x}^{\delta_{2} \delta_{3}^{\prime}}
\end{array}\right)=A\left[e^{-i \alpha}\left(C_{\delta}^{2 \dagger} C_{\delta^{\prime}}^{1}-C_{\delta}^{3 \dagger} C_{\delta^{\prime}}^{4}\right)+e^{i \alpha}\left(C_{\delta}^{1 \dagger} C_{\delta^{\prime}}^{2}-C_{\delta}^{4 \dagger} C_{\delta^{\prime}}^{3}\right)\right], } \\
{\left[v_{z}\right]=} & \left(\begin{array}{ccc}
v_{z}^{\delta_{1} \delta_{1}^{\prime}} & v_{z}^{\delta_{1} \delta_{2}^{\prime}} & v_{z}^{\delta_{1} \delta_{3}^{\prime}} \\
v_{z}^{\delta_{2} \delta_{1}^{\prime}} & v_{z}^{\delta_{2} \delta_{2}^{\prime}} & v_{z}^{\delta_{2} \delta_{3}^{\prime}}
\end{array}\right)=i A\left[e^{-i \alpha}\left(C_{\delta}^{2 \dagger} C_{\delta^{\prime}}^{1}-C_{\delta}^{3 \dagger} C_{\delta^{\prime}}^{4}\right)+e^{i \alpha}\left(C_{\delta}^{4 \dagger} C_{\delta^{\prime}}^{3}-C_{\delta}^{1 \dagger} C_{\delta^{\prime}}^{2}\right)\right] \\
& +\left[U_{+-+-} C_{\delta, m v^{\prime}}^{1 \dagger} R C_{\delta^{\prime}, n v}^{1}+U_{+--+} C_{\delta, m v^{\prime}}^{2 \dagger} R C_{\delta^{\prime}, n v}^{2}+U_{+-+-} C_{\delta, m v^{\prime}}^{3 \dagger} R C_{\delta^{\prime}, n v}^{3}+U_{+--+} C_{\delta, m v^{\prime}}^{4 \dagger} R C_{\delta^{\prime}, n v}^{4}\right],
\end{aligned}
$$

where $R$ is a $9 \times 9$ matrix with its element is

$$
R_{m n}=\left\{\begin{array}{cc}
0, & m=n \\
\frac{2 i\left[-1+(-1)^{m+n}\right] m n \sin 2 \theta}{L\left(m^{2}-n^{2}\right)} \mathbf{I}_{3} & m \neq n .
\end{array}\right.
$$


Now define

$$
C_{\delta}^{1,2,3,4}=\left(\begin{array}{ll}
C_{\delta_{1}, 10}^{1,2,3,4} & C_{\delta_{2}, 10}^{1,2,3,4} \\
C_{\delta_{1}, 11}^{1,2,3,4} & C_{\delta_{2}, 11}^{1,2,3,4} \\
C_{\delta_{1}, 12}^{1,2,3,4} & C_{\delta_{2}, 12}^{1,2,3,4} \\
C_{\delta_{1}, 20}^{1,2,3,4} & C_{\delta_{2}, 20}^{1,2,3,4} \\
C_{\delta_{1}, 21}^{1,2,3,4} & C_{\delta_{2}, 21}^{1,2,3,4} \\
C_{\delta_{1}, 22}^{1,2,3,4} & C_{\delta_{2}, 22}^{1,2,3,4} \\
C_{\delta_{1}, 30}^{1,2,3,4} & C_{\delta_{2}, 30}^{1,2,3,4} \\
C_{\delta_{1}, 31}^{1,2,3,4} & C_{\delta_{2}, 31}^{1,2,3,4}
\end{array}\right), \quad C_{\delta^{\prime}}^{1,2,3,4}=\left(\begin{array}{lll}
C_{\delta_{1}^{\prime}, 11}^{1,2,3,4} & C_{\delta_{2}^{\prime}, 11}^{1,2,3,4} & C_{\delta_{3}^{\prime}, 11}^{1,2,3,4} \\
C_{\delta_{1}^{\prime}, 12}^{1,2,3,4} & C_{\delta_{2}^{\prime}, 12}^{1,2,3,4} & C_{\delta_{3}^{\prime}, 12}^{1,2,3,4} \\
C_{\delta_{1}^{\prime}, 20}^{1,2,3,4} & C_{\delta_{2}^{\prime}, 20}^{1,2,3,4} & C_{\delta_{3}^{\prime}, 20}^{1,2,3,4} \\
C_{\delta_{1}^{\prime}, 21}^{1,2,3,4} & C_{\delta_{2}^{\prime}, 21}^{1,2,3,4} & C_{\delta_{3}^{\prime}, 21}^{1,2,3,4} \\
C_{\delta_{1}^{\prime}, 22}^{1,2,3,4} & C_{\delta_{2}^{\prime}, 22}^{1,2,3,4} & C_{\delta_{3}^{\prime}, 22}^{1,2,3,4} \\
C_{\delta_{1}^{\prime}, 30}^{1,2,3,4} & C_{\delta_{2}^{\prime}, 30}^{1,2,3,4} & C_{\delta_{3}^{\prime}, 30}^{1,2,3,4} \\
C_{\delta_{1}^{\prime}, 31}^{1,2,3,4} & C_{\delta_{2}^{\prime}, 31}^{1,2,3,4} & C_{\delta_{3}^{\prime}, 31}^{1,2,3,4} \\
C_{\delta_{1}^{\prime}, 32}^{1,2,3,4} & C_{\delta_{2}^{\prime}, 32}^{1,2,3,4} & C_{\delta_{3}^{\prime}, 32}^{1,2,3,4}
\end{array}\right) .
$$

Then

$$
\begin{aligned}
{\left[v_{x, a}\right]=\frac{\sqrt{2}}{\ell_{y^{\prime}}}\left[\left(C_{2}+M_{2}\right)\left(C_{\delta}^{1 \dagger} V C_{\delta^{\prime}}^{1}+C_{\delta}^{3 \dagger} V C_{\delta^{\prime}}^{3}\right)+\left(C_{2}-M_{2}\right)\left(C_{\delta}^{2 \dagger} V C_{\delta^{\prime}}^{2}+C_{\delta}^{4 \dagger} V C_{\delta^{\prime}}^{4}\right)\right], } \\
{\left[v_{z, a}\right]=\frac{i}{\sqrt{2} \ell_{y^{\prime}} t_{y^{\prime}}}\left[\left(U_{++++}+U_{+-+-} \cos 2 \theta\right) C_{\delta}^{1 \dagger} V C_{\delta^{\prime}}^{1}+\left(U_{++--}+U_{+--+} \cos 2 \theta\right) C_{\delta}^{2 \dagger} V C_{\delta^{\prime}}^{2}\right.} \\
\left.+\left(U_{++++}+U_{+-+-} \cos 2 \theta\right) C_{\delta}^{3 \dagger} V C_{\delta^{\prime}}^{3}+\left(U_{++--}+U_{+--+} \cos 2 \theta\right) C_{\delta}^{4 \dagger} V C_{\delta^{\prime}}^{4}\right],
\end{aligned}
$$

where $\mathrm{V}$ is a diagonal matrix having the form

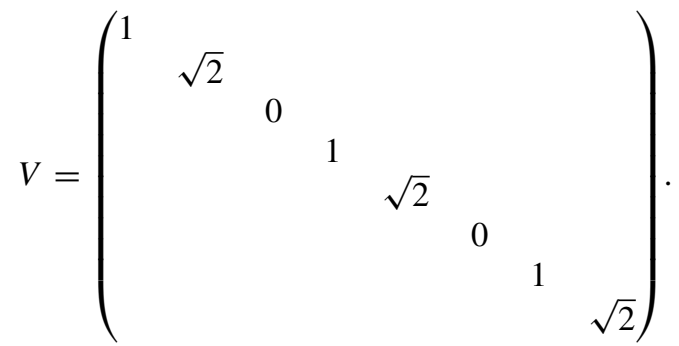

We continue to define

$$
C_{\delta}^{1,2,3,4}=\left(\begin{array}{ll}
C_{\delta_{1}, 11}^{1,2,3,4} & C_{\delta_{2}, 11}^{1,2,3,4} \\
C_{\delta_{1}, 12}^{1,2,3,4} & C_{\delta_{2}, 12}^{1,2,3,4} \\
C_{\delta_{1}, 20}^{1,2,3,4} & C_{\delta_{2}, 20}^{1,2,3,4} \\
C_{\delta_{1}, 21}^{1,2,3,4} & C_{\delta_{2}, 21}^{1,2,3,4} \\
C_{\delta_{1}, 22}^{1,2,3,4} & C_{\delta_{2}, 22}^{1,2,3,4} \\
C_{\delta_{1}, 30}^{1,2,3,4} & C_{\delta_{2}, 30}^{1,2,3,4} \\
C_{\delta_{1}, 2,31}^{1,2,3} & C_{\delta_{2}, 31}^{1,2,3,4} \\
C_{\delta_{1}, 2,3,4}^{1,2,3} & C_{\delta_{2}, 32}^{1,2,3,4}
\end{array}\right), \quad C_{\delta^{\prime}}^{1,2,3,4}=\left(\begin{array}{lll}
C_{\delta_{1}^{\prime}, 10}^{1,2,3,4} & C_{\delta_{2}^{\prime}, 10}^{1,2,3,4} & C_{\delta_{3}^{\prime}, 10}^{1,2,3,4} \\
C_{\delta_{1}^{\prime}, 11}^{1,2,3,4} & C_{\delta_{2}^{\prime}, 11}^{1,2,3,4} & C_{\delta_{3}^{\prime}, 11}^{1,2,3,4} \\
C_{\delta_{1}^{\prime}, 12}^{1,2,3,4} & C_{\delta_{2}^{\prime}, 12}^{1,2,3,4} & C_{\delta_{3}^{\prime}, 12}^{1,2,3,4} \\
C_{\delta_{1}^{\prime}, 20}^{1,2,3,4} & C_{\delta_{2}^{\prime}, 20}^{1,2,3,4} & C_{\delta_{3}^{\prime}, 20}^{1,2,3,4} \\
C_{\delta_{1}^{\prime}, 21}^{1,2,3,4} & C_{\delta_{2}^{\prime}, 21}^{1,2,3,4} & C_{\delta_{3}^{\prime}, 21}^{1,2,3} \\
C_{\delta_{1}^{\prime}, 22}^{1,2,3,4} & C_{\delta_{2}^{\prime}, 22}^{1,2,3,4} & C_{\delta_{3}^{\prime}, 22}^{1,2,3,4} \\
C_{\delta_{1}^{\prime}, 30}^{1,2,3,4} & C_{\delta_{2}^{\prime}, 30}^{1,2,3,4} & C_{\delta_{3}^{\prime}, 30}^{1,2,3,4} \\
C_{\delta_{1}^{\prime}, 31}^{1,2,3,4} & C_{\delta_{2}^{\prime}, 31}^{1,2,3,4} & C_{\delta_{3}^{\prime}, 31}^{1,2,3,4}
\end{array}\right) .
$$

Then

$$
\begin{gathered}
{\left[v_{x, a^{\dagger}}\right]=\frac{\sqrt{2}}{\ell_{y^{\prime}}}\left[\left(C_{2}+M_{2}\right)\left(C_{\delta}^{1 \dagger} V C_{\delta^{\prime}}^{1}+C_{\delta}^{3 \dagger} V C_{\delta^{\prime}}^{3}\right)+\left(C_{2}-M_{2}\right)\left(C_{\delta}^{2 \dagger} V C_{\delta^{\prime}}^{2}+C_{\delta}^{4 \dagger} V C_{\delta^{\prime}}^{4}\right)\right],} \\
{\left[v_{z, a^{\dagger}}\right]=-\frac{i}{\sqrt{2} \ell_{y^{\prime}} t_{y^{\prime}}}\left[\left(U_{++++}+U_{+-+-} \cos 2 \theta\right) C_{\delta}^{1 \dagger} V C_{\delta^{\prime}}^{1}+\left(U_{++--}+U_{+--+} \cos 2 \theta\right) C_{\delta}^{2 \dagger} V C_{\delta^{\prime}}^{2}\right.} \\
\left.+\left(U_{++++}+U_{+-+-} \cos 2 \theta\right) C_{\delta}^{3 \dagger} V C_{\delta^{\prime}}^{3}+\left(U_{++--}+U_{+--+} \cos 2 \theta\right) C_{\delta}^{4 \dagger} V C_{\delta^{\prime}}^{4}\right] .
\end{gathered}
$$

Therefore, the total velocity matrix is given by

$$
\begin{aligned}
& {\left[v_{x}\right]=\left[v_{x}\right]+\left[v_{x, a}\right]+\left[v_{x, a^{\dagger}}\right],} \\
& {\left[v_{z}\right]=\left[v_{z}\right]+\left[v_{z, a}\right]+\left[v_{z, a^{\dagger}}\right] .}
\end{aligned}
$$



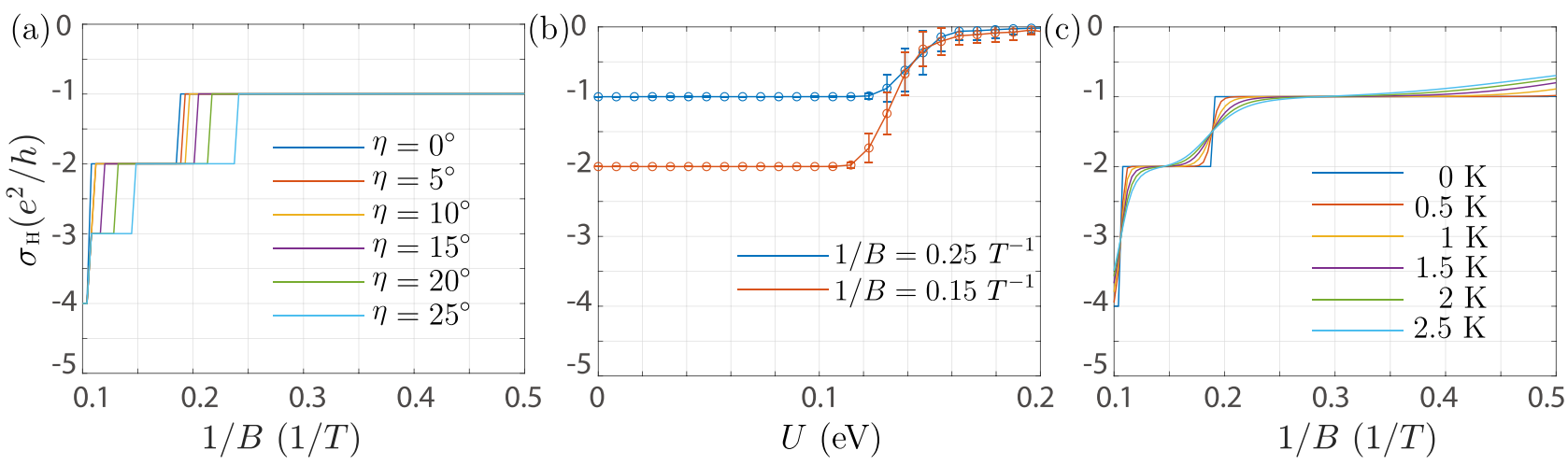

FIG. 9. The Hall conductance $\sigma_{\mathrm{H}}$ as a function of (a) the inverse of magnetic field $1 / B$ at different tilting angles of the magnetic field, (b) disorder strength $U$ at different magnetic field strengths, and (c) the inverse of magnetic field $1 / B$ at different temperatures. In (b), the error bars show the standard deviation averaged over 200 samples. The thicknesses of the [001]-direction slabs are $100 \mathrm{~nm}$.

We define the energy-dependent matrix as

$$
[E]=\left(\begin{array}{lll}
\left(E_{\delta_{1}}-E_{\delta_{1}^{\prime}}\right)^{2}+\Gamma^{2} & \left(E_{\delta_{1}}-E_{\delta_{2}^{\prime}}\right)^{2}+\Gamma^{2} & \left(E_{\delta_{1}}-E_{\delta^{\prime}}\right)^{2}+\Gamma^{2} \\
\left(E_{\delta_{2}}-E_{\delta_{1}^{\prime}}\right)^{2}+\Gamma^{2} & \left(E_{\delta_{2}}-E_{\delta_{2}^{\prime}}\right)^{2}+\Gamma^{2} & \left(E_{\delta_{2}}-E_{\delta_{3}^{\prime}}\right)^{2}+\Gamma^{2}
\end{array}\right) .
$$

Finally, the total Hall conductivity at zero temperature is given by

$$
\sigma_{\mathrm{H}}=t_{y^{\prime}} \frac{e^{2}}{h} \frac{4}{L \ell_{y^{\prime}}^{4}} \sum_{l j} \frac{\left[v_{x}\right]_{l j}\left[v_{z}\right]_{l j}^{*}}{[E]_{l j}} .
$$

Figure 9(a) shows the Hall conductance $\sigma_{\mathrm{H}}$ as a function of $1 / B$ at different tilting angles of the magnetic field $\eta$, where we consider both the orbital effect (Landau levels) and Zeeman effect of a tilted magnetic field $\mathbf{B}=\left(0, B_{y}, B_{z}=B_{y} \tan \eta\right)$. Using the Landau gauge, the vector potential of the magnetic field $\mathbf{A}=\left(B_{y} z-B_{z} y, 0,0\right)$, where $\mathbf{B}=\nabla \times \mathbf{A}$. Figure 9 (a) shows that the Hall conductance plateaus originated from the Zeeman splitting mechanism of the helical edge states are robust against the change in the magnetic field direction, except that the positions of the conductance plateaus are shifted.

Figure 9(b) shows the Hall conductance $\sigma_{\mathrm{H}}$ as a function of disorder strength $U$ at different magnetic field strengths. The disorder-averaged Hall conductance is obtained by numerically calculated the transport in a four-terminal device by using the Landauer-Büttiker formula [53-55] and the recursive Green's function method [56,57]. We adopt the Anderson-type disorder by considering random on-site energies fluctuating in the energy interval $[-U, U]$, where $U$ is the disorder strength. With increasing disorder strength, the conductance remains quantized until the disorder strength $U$ exceeds $100 \mathrm{meV}$, which is much larger compared to the confinement effect induced gap (about $5 \mathrm{meV}$ ). Therefore, we show that Hall plateaus are robust against weak disorder.

Figure 9(c) shows the Hall conductance $\sigma_{\mathrm{H}}$ as a function of $1 / B$ at different temperatures. The Hall plateaus are robust at low temperatures accessible to experiments, but show a tendency of being destroyed at higher temperatures. We treat the temperature effect as the broadening of the Fermi surface. At higher temperatures, other effects such as inelastic scattering induced by phonons need to be considered as well and could be studied in the future.

In Fig. 10, we plot $\sigma_{\mathrm{H}}$ as a function of $1 / B$ with different Fermi levels for the [110]-direction slab of the Dirac semimetal. For the case without the Zeeman effect, $\sigma_{\mathrm{H}}$ is always an even number due to the spin degeneration. The shift of the plateaus for different thicknesses obeys the modified Lifshitz-Onsager relation [31,33]. No shift occurs for $E_{\mathrm{F}}=0$. Therefore, we conclude that the phase shift for $E_{\mathrm{F}}=0$ originates from the Zeeman effect. While for $E_{\mathrm{F}} \neq 0$, the phase shift is contributed simultaneously by the Zeeman effect and the Weyl orbit.

\section{APPENDIX D: TRANSPORT}

We will investigate the transport properties of the system by using the Landauer-Büttiker-Fisher-Lee formula [53-55] and the recursive Green's function method [56,57]. The linear conductance can be obtained by the transmission coefficient $T_{p q}$ from the terminal $p$ to terminal $q$, where $T_{p q}=\operatorname{Tr}\left[\Gamma_{p} G^{r} \Gamma_{q} G^{a}\right]$ is the transmission coefficient. The linewidth function $\Gamma_{p}(\mu)=$ $i\left[\Sigma_{p}^{r}-\Sigma_{p}^{a}\right]$ with $\Sigma_{p}^{r / a}$ is the retarded/advanced self-energy at the terminal $p$, and the Green's functions $G^{r / a}$ are calculated from $G^{r}=\left(G^{a}\right)^{\dagger}=\left[E_{f} I-H_{C}-\sum_{p} \Sigma_{p}^{r}\right]^{-1}$, where $E_{f}$ is the Fermi energy and $H_{C}$ is the Hamiltonian matrix of the central scattering region.

We adopt a cubic lattice as the central scattering device with the side length $L_{x, y, z}$, and semi-infinite square lattice as the leads connected to the device. The Hamiltonian for the semi-infinite lead is $H_{L}=\sum_{i \alpha} \mu_{L} c_{i \alpha}^{\dagger} c_{i \alpha}+\sum_{\langle i \alpha, j \alpha} t c_{i \alpha}^{\dagger} c_{j \alpha}$, where $\mu_{L}$ is the chemical potential of the leads. In modeling the leads, we only consider the nearest-neighbor hopping of the lattice. 

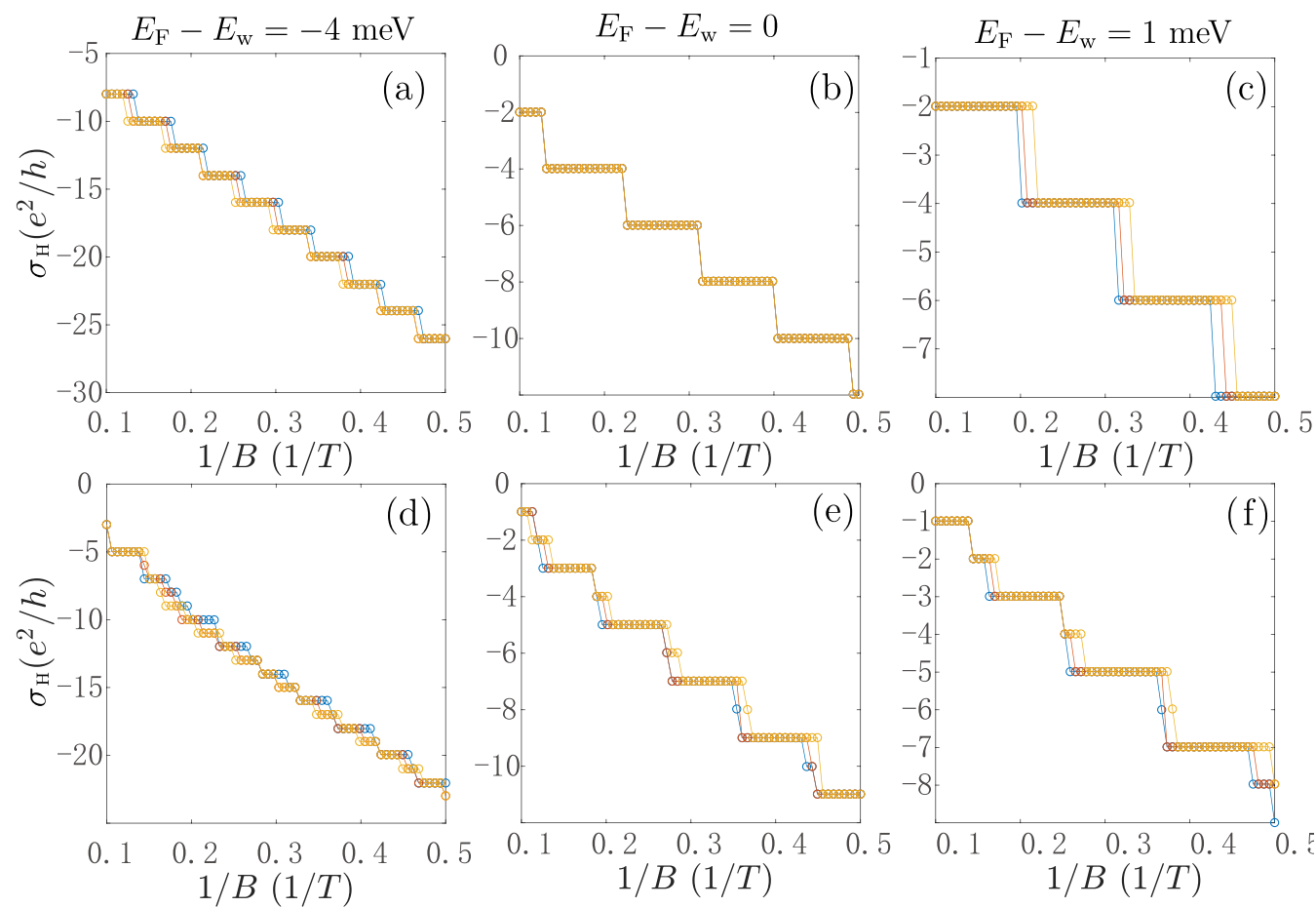

FIG. 10. The Hall conductance $\sigma_{\mathrm{H}}$ as a function of the inverse of magnetic field $1 / B$ with the Fermi energy $(\mathrm{a}, \mathrm{d}) E_{\mathrm{F}}=-0.004 \mathrm{eV},(\mathrm{b}, \mathrm{e})$ $E_{\mathrm{F}}=0$, and (c,f) $E_{\mathrm{F}}=0.001 \mathrm{eV}$. The thicknesses of the [110]-direction slabs are 80 (red), 90 (blue), and $100 \mathrm{~nm}$ (yellow), respectively. The Zeeman terms are excluded and included in the upper and lower panels, respectively.

Because of time-reversal symmetry, a Dirac semimetal can be regarded as a Weyl semimetal and its time reversal. On the other hand, as the calculation for the transport is computationally very demanding, we will numerically calculate the transport of Weyl semimetal. We also find that the transport of Dirac semimetals shows similar properties to the case of Weyl semimetals.

\section{Weyl semimetals}

We will consider the following Hamiltonian [6,13]:

$$
H_{0}=D_{1} k_{y}^{2}+D_{2}\left(k_{x}^{2}+k_{z}^{2}\right)+A\left(k_{x} \sigma_{x}+k_{y} \sigma_{y}\right)+M\left(k_{w}^{2}-k^{2}\right) \sigma_{z},
$$

which hosts two Weyl nodes at $\left(0,0, \pm k_{w}\right)$ with energy $E_{w}=D_{2} k_{w}^{2}$. To obtain the dispersion of the Weyl semimetal slab along an arbitrary growth direction, we rotate the $y$ axis to the $y^{\prime}$ axis through the rotation matrix in Eq. (2). The [010], and [001] directions correspond to $(\alpha, \theta)=(0,0)$ and $(\pi / 2,0)$, respectively. We choose $\mathbf{A}=\left(B_{y^{\prime}} z, 0,0\right)$, the corresponding magnetic field is $\mathbf{B}=\left(0, B_{y^{\prime}}, 0\right)$. The wave vector should be replaced with the Peierls transformation

$$
\begin{aligned}
\mathbf{k} & =\left(k_{x^{\prime}}, k_{y^{\prime}}, k_{z^{\prime}}\right) \rightarrow \mathbf{k}^{\prime}+\frac{\mathbf{e}}{\mathbf{h}} \mathbf{A}=\left(k_{x^{\prime}}+\frac{e}{\hbar} B_{y^{\prime}} z^{\prime},-i \partial_{y^{\prime}},-i \partial_{z^{\prime}}\right), \\
& =\left(k_{x^{\prime}}+\frac{z^{\prime}}{\ell_{y^{\prime}}^{2}},-i \partial_{y^{\prime}},-i \partial_{z^{\prime}}\right),
\end{aligned}
$$

with $\ell_{y^{\prime}}=\sqrt{\hbar /\left|e B_{y^{\prime}}\right|}$. After the Fourier transformation, we obtain the tight-bing model in the real space

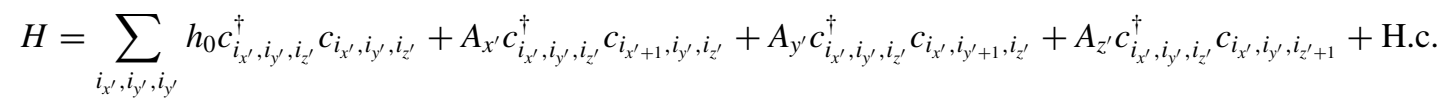

Here, the parameters are

$$
\begin{aligned}
h_{0} & =\left(2 D_{1}+4 D_{2}\right) \sigma_{0}+\left(k_{w}^{2}-6\right) M \sigma_{z}, \\
A_{x^{\prime}} & =-D_{2} \sigma_{0}-i A \sigma_{x} / 2+M \sigma_{z}, \\
A_{y^{\prime}} & =-D_{1} \sigma_{0}-i A \sigma_{y} / 2+M \sigma_{z}, \\
A_{z^{\prime}} & =-D_{2} \sigma_{0}+M \sigma_{z},
\end{aligned}
$$



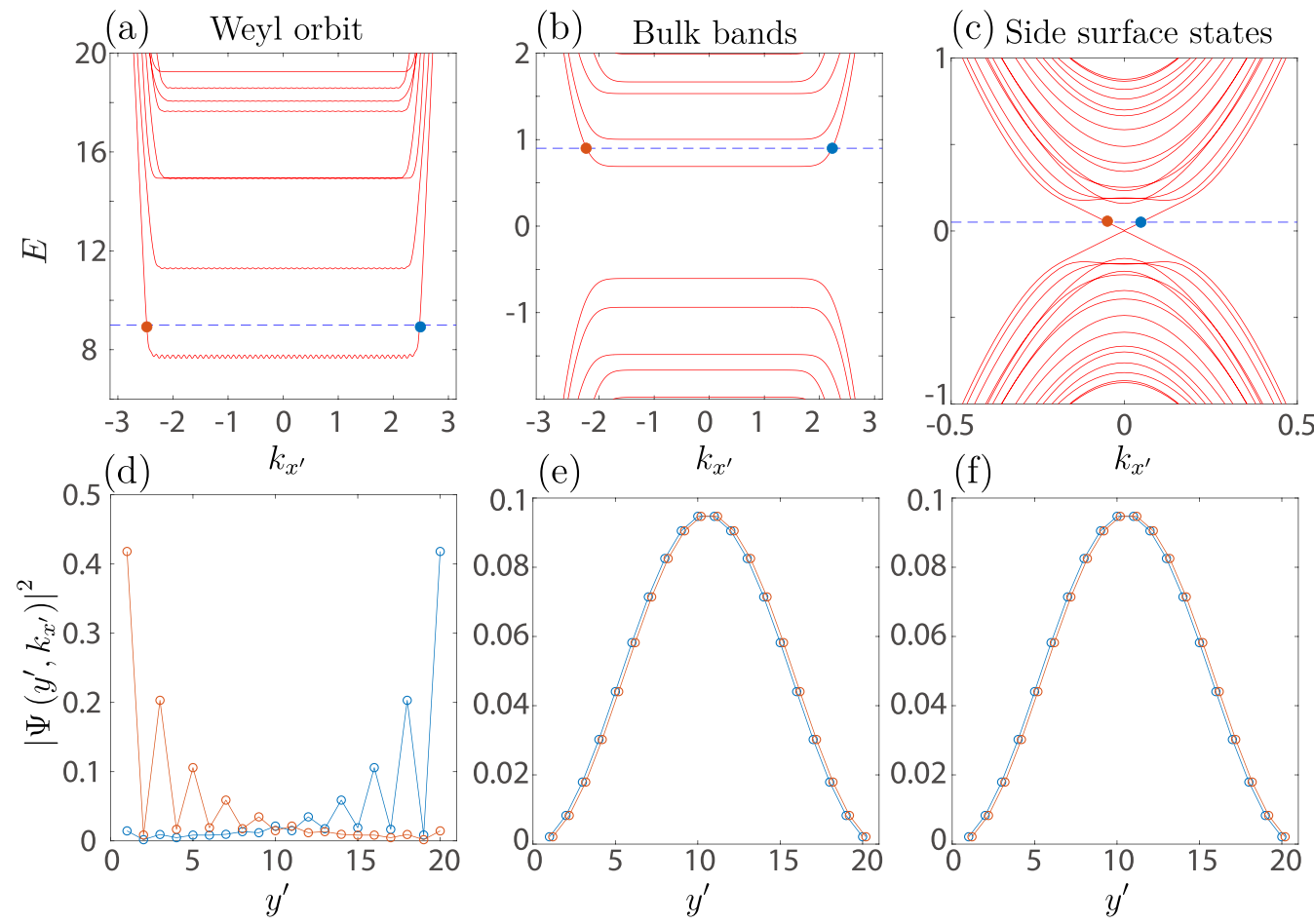

FIG. 11. (a)-(c) Energy spectra of Weyl-semimetal films with open boundary conditions along the $y^{\prime}$ and $z^{\prime}$ directions and periodic boundary condition along the $x^{\prime}$ direction. Here, the sizes in the $y^{\prime}$ and $z^{\prime}$ directions are taken as $L_{y^{\prime}}=20 a_{y^{\prime}}$ and $L_{z^{\prime}}=40 a_{z^{\prime}}$. The red curves are the bulk bands and the blue dashed curves label the Fermi levels. The parameters are (a) $(\alpha, \theta)=(0,0), M=5, A=50, D_{2}=4, D_{1}=1$, $B_{y}=1 / 10$, and $k_{w}=1.5$ [13]; (b) $(\pi / 2,0), M=5, A=1, B_{y}=1 / 10$ and $D_{1}=D_{2}=k_{w}=0$; and (c) $(\pi / 2,0), M=5, A=1, D_{1}=D_{2}$, $B_{y}=0$, and $k_{w}=0.25$. (d)-(f) The wave function distributions along the $y^{\prime}$ direction. The red and blue dot lines correspond the red and blue points shown in (a)-(c). Here we define $\left|\Psi\left(k_{x^{\prime}}, y^{\prime}\right)\right|^{2}=\sum_{z^{\prime}}\left|\psi\left(k_{x^{\prime}}, y^{\prime}, z^{\prime}\right)\right|^{2}$.

and

$$
\begin{aligned}
h_{0} & =\left(2 D_{1}+4 D_{2}\right) \sigma_{0}+\left(k_{w}^{2}-6\right) M \sigma_{z}, \\
A_{x^{\prime}} & =-D_{2} \sigma_{0}-i A \sigma_{x} / 2+M \sigma_{z}, \\
A_{y^{\prime}} & =-D_{2} \sigma_{0}+M \sigma_{z}, \\
A_{z^{\prime}} & =-D_{1} \sigma_{0}-i A \sigma_{y} / 2+M \sigma_{z},
\end{aligned}
$$

for the [010] and [001] directions, respectively. When the magnetic field is introduced, according to the Peierls substitution, we replace

$$
c_{i_{x^{\prime}}, i_{y^{\prime}}, i_{z^{\prime}}}^{\dagger} c_{i_{x^{\prime}}+d_{x^{\prime}}, i_{y^{\prime}}, i_{z^{\prime}}} \rightarrow \frac{2 \pi d a_{x^{\prime}} a_{z^{\prime}} B_{y^{\prime}} i_{z^{\prime}}}{\Phi_{0}},
$$

where $d_{x^{\prime}}= \pm 1, a_{x^{\prime}, y^{\prime}, z^{\prime}}=1$ are the lattice constants, and $\Phi_{0}=h / e$ is the magnetic flux quantum.

As shown in Fig. 11, to make a comparative study, we study the spectra and wave-function distributions of three different systems hosting the quantized Hall conductance: (i) the Landau levels in Fig. 11(a) originate from the Weyl orbit, and the wave-function distributions [Fig. 11(d)] are mainly located at the top and bottom surfaces along the $y^{\prime}$ direction, (ii) the Landau levels in Fig. 11(b) originate from confinement induced bulk subbands, and the wave-function distributions [Fig. 11(e)] are mainly located at the central region along the $y^{\prime}$ direction, (iii) the states in Fig. 11(c) originate from the side surface states, which are mainly located on the boundary on the $x^{\prime}-z^{\prime}$ plane, and at the central region along the $y^{\prime}$ direction [Fig. 11(f)].

\section{Dirac semimetals}

In Sec. IV, we show that the thickness dependent conductance plateaus of Dirac semimetals can be attributed to various reasons. A Dirac semimetal can be regarded as a Weyl semimetal and its time reversal. Therefore, the above derivations for Weyl semimetals also apply to Dirac semimetals. Here, we propose that the signatures of the quantum Hall effect induced by the Weyl orbit are the surface Hall conductance plateaus by using the device shown in the main text. The quantized Hall conductance induced by the mixing of the Weyl orbit can also be identified by using same device. Furthermore, the quantized Hall conductance induced by the side surface states can be identified by measuring the nonlocal resistance [51]. 
[1] K. V. Klitzing, G. Dorda, and M. Pepper, New Method for High-Accuracy Determination of the Fine-Structure Constant Based on Quantized Hall Resistance, Phys. Rev. Lett. 45, 494 (1980).

[2] D. J. Thouless, M. Kohmoto, M. P. Nightingale, and M. den Nijs, Quantized Hall Conductance in a Two-Dimensional Periodic Potential, Phys. Rev. Lett. 49, 405 (1982).

[3] B. I. Halperin, Possible states for a three-dimensional electron gas in a strong magnetic field, Jpn. J. Appl. Phys. 26, 1913 (1987).

[4] S.-C. Zhang and J. Hu, A four-dimensional generalization of the quantum Hall effect, Science 294, 823 (2001).

[5] O. Zilberberg, S. Huang, J. Guglielmon, M. Wang, K. P. Chen, Y. E. Kraus, and M. C. Rechtsman, Photonic topological boundary pumping as a probe of $4 \mathrm{D}$ quantum Hall physics, Nature (London) 553, 59 (2018).

[6] C. M. Wang, H.-P. Sun, H.-Z. Lu, and X. C. Xie, 3D Quantum Hall Effect of Fermi Arcs in Topological Semimetals, Phys. Rev. Lett. 119, 136806 (2017).

[7] H.-Z. Lu, 3D quantum Hall effect, Natl. Sci. Rev. 6, 208 (2018).

[8] R. Chen, T. Liu, C. M. Wang, H.-Z. Lu, and X. C. Xie, FieldTunable One-Sided Higher-Order Topological Hinge States in Dirac Semimetals, Phys. Rev. Lett. 127, 066801 (2021).

[9] F. Qin, S. Li, Z. Z. Du, C. M. Wang, W. Zhang, D. Yu, H.-Z. Lu, and X. C. Xie, Theory for the Charge-Density-Wave Mechanism of 3D Quantum Hall Effect, Phys. Rev. Lett. 125, 206601 (2020).

[10] M. Chang and L. Sheng, Three-dimensional quantum Hall effect in the excitonic phase of a Weyl semimetal, Phys. Rev. B 103, 245409 (2021).

[11] M. Chang, H. Geng, L. Sheng, and D. Y. Xing, Threedimensional quantum Hall effect in Weyl semimetals, Phys. Rev. B 103, 245434 (2021).

[12] D.-H.-M. Nguyen, K. Kobayashi, J.-E. R. Wichmann, and K. Nomura, Quantum Hall effect induced by chiral Landau levels in topological semimetal films, Phys. Rev. B 104, 045302 (2021).

[13] H. Li, H. Liu, H. Jiang, and X. C. Xie, 3D Quantum Hall Effect and a Global Picture of Edge States in Weyl Semimetals, Phys. Rev. Lett. 125, 036602 (2020)

[14] R. Yu, W. Zhang, H.-J. Zhang, S.-C. Zhang, X. Dai, and Z. Fang, Quantized anomalous Hall effect in magnetic topological insulators, Science 329, 61 (2010).

[15] C.-Z. Chang, J. Zhang, X. Feng, J. Shen, Z. Zhang, M. Guo et al., Experimental observation of the quantum anomalous Hall effect in a magnetic topological insulator, Science 340, 167 (2013).

[16] I. Sodemann and L. Fu, Quantum Nonlinear Hall Effect Induced by Berry Curvature Dipole in Time-Reversal Invariant Materials, Phys. Rev. Lett. 115, 216806 (2015).

[17] T. Low, Y. Jiang, and F. Guinea, Topological currents in black phosphorus with broken inversion symmetry, Phys. Rev. B 92, 235447 (2015).

[18] Q. Ma, S.-Y. Xu, H. Shen, D. MacNeill, V. Fatemi, T.-R. Chang et al., Observation of the nonlinear Hall effect under time-reversal-symmetric conditions, Nature (London) 565, 337 (2019).

[19] K. Kang, T. Li, E. Sohn, J. Shan, and K. F. Mak, Observation of the nonlinear anomalous Hall effect in $2 \mathrm{D} \mathrm{WTe}{ }_{2}$, Nat. Mater. 18, 324 (2019).
[20] Z. Z. Du, C. M. Wang, H.-Z. Lu, and X. C. Xie, Band Signatures for Strong Nonlinear Hall Effect in Bilayer $\mathrm{WTe}_{2}$, Phys. Rev. Lett. 121, 266601 (2018).

[21] Z. Z. Du, C. M. Wang, S. Li, H.-Z. Lu, and X. C. Xie, Disorderinduced nonlinear Hall effect with time-reversal symmetry, Nat. Commun. 10, 3047 (2019).

[22] C. Zhang, A. Narayan, S. Lu, J. Zhang, H. Zhang, Z. Ni et al., Evolution of Weyl orbit and quantum Hall effect in Dirac semimetal $\mathrm{Cd}_{3} \mathrm{As}_{2}$, Nat. Commun. 8, 1272 (2017).

[23] C. Zhang, Y. Zhang, X. Yuan, S. Lu, J. Zhang, A. Narayan et al., Quantum Hall effect based on Weyl orbit in $\mathrm{Cd}_{3} \mathrm{As}_{2}$, Nature (London) 565, 331 (2019).

[24] S. Nishihaya, M. Uchida, Y. Nakazawa, R. Kurihara, K. Akiba, M. Kriener, A. Miyake, Y. Taguchi, M. Tokunaga, and M. Kawasaki, Quantized surface transport in topological Dirac semimetal films, Nat. Commun. 10, 2564 (2019).

[25] B.-C. Lin, S. Wang, S. Wiedmann, J.-M. Lu, W.-Z. Zheng, D. $\mathrm{Yu}$, and Z.-M. Liao, Observation of an Odd-Integer Quantum Hall Effect from Topological Surface States in $\mathrm{Cd}_{3} \mathrm{As}_{2}$, Phys. Rev. Lett. 122, 036602 (2019).

[26] T. Schumann, L. Galletti, D. A. Kealhofer, H. Kim, M. Goyal, and S. Stemmer, Observation of the Quantum Hall Effect in Confined Films of the Three-Dimensional Dirac Semimetal $\mathrm{Cd}_{3}$ $\mathrm{As}_{2}$, Phys. Rev. Lett. 120, 016801 (2018).

[27] L. Galletti, T. Schumann, O. F. Shoron, M. Goyal, D. A Kealhofer, H. Kim, and S. Stemmer, Two-dimensional Dirac fermions in thin films of $\mathrm{Cd}_{3} \mathrm{As}_{2}$, Phys. Rev. B 97, 115132 (2018).

[28] M. Uchida, Y. Nakazawa, S. Nishihaya, K. Akiba, M. Kriener, Y. Kozuka et al., Quantum Hall states observed in thin films of Dirac semimetal $\mathrm{Cd}_{3} \mathrm{As}_{2}$, Nat. Commun. 8, 2274 (2017).

[29] S. Nishihaya, M. Uchida, Y. Nakazawa, M. Kriener, Y. Kozuka, Y. Taguchi, and M. Kawasaki, Gate-tuned quantum Hall states in Dirac semimetal $\left(\mathrm{Cd}_{1-x} \mathrm{Zn}_{x}\right) \mathrm{As}_{2}$, Sci. Adv. 4, eaar5668 (2018).

[30] D. A. Kealhofer, L. Galletti, T. Schumann, A. Suslov, and S. Stemmer, Topological Insulator State and Collapse of the Quantum Hall Effect in a Three-Dimensional Dirac Semimetal Heterojunction, Phys. Rev. X 10, 011050 (2020).

[31] A. C. Potter, I. Kimchi, and A. Vishwanath, Quantum oscillations from surface Fermi arcs in Weyl and Dirac semimetals, Nat. Commun. 5, 5161 (2014).

[32] P. J. W. Moll, N. L. Nair, T. Helm, A. C. Potter, I. Kimchi, A. Vishwanath, and J. G. Analytis, Transport evidence for Fermiarc-mediated chirality transfer in the Dirac semimetal $\mathrm{Cd}_{3} \mathrm{As}_{2}$, Nature (London) 535, 266 (2016).

[33] Y. Zhang, D. Bulmash, P. Hosur, A. C. Potter, and A. Vishwanath, Quantum oscillations from generic surface Fermi arcs and bulk chiral modes in Weyl semimetals, Sci. Rep. 6, 23741 (2016).

[34] C. Zhang, Y. Zhang, H.-Z. Lu, X. C. Xie, and F. Xiu, Cycling Fermi arc electrons with Weyl orbits, Nat. Rev. Phys. 3, 660 (2021).

[35] Z. Wang, H. Weng, Q. Wu, X. Dai, and Z. Fang, Threedimensional Dirac semimetal and quantum transport in $\mathrm{Cd}_{3} \mathrm{As}_{2}$, Phys. Rev. B 88, 125427 (2013).

[36] J. Cano, B. Bradlyn, Z. Wang, M. Hirschberger, N. P. Ong, and B. A. Bernevig, Chiral anomaly factory: Creating Weyl fermions with a magnetic field, Phys. Rev. B 95, 161306(R) (2017). 
[37] T. Schumann, M. Goyal, H. Kim, and S. Stemmer, Molecular beam epitaxy of $\mathrm{Cd}_{3} \mathrm{As}_{2}$ on a III-V substrate, APL Mater. 4, 126110 (2016).

[38] S. Jeon, B. B. Zhou, A. Gyenis, B. E. Feldman, I. Kimchi, A. C. Potter, Q. D. Gibson, R. J. Cava, A. Vishwanath, and A. Yazdani, Landau quantization and quasiparticle interference in the three-dimensional Dirac semimetal $\mathrm{Cd}_{3} \mathrm{As}_{2}$, Nat. Mater. 13, 851 (2014)

[39] S. B. Zhang, Y. Y. Zhang, and S. Q. Shen, Robustness of quantum spin Hall effect in an external magnetic field, Phys. Rev. B 90, 115305 (2014).

[40] X. Xiao, S. A. Yang, Z. Liu, H. Li, and G. Zhou, Anisotropic quantum confinement effect and electric control of surface states in Dirac semimetal nanostructures, Sci. Rep. 5, 7898 (2015).

[41] H. Pan, M. Wu, Y. Liu, and S. A. Yang, Electric control of topological phase transitions in Dirac semimetal thin films, Sci. Rep. 5, 14639 (2015).

[42] R. Chen, D.-H. Xu, and B. Zhou, Topological Anderson insulator phase in a Dirac-semimetal thin film, Phys. Rev. B 95, 245305 (2017).

[43] X.-L. Qi and S.-C. Zhang, Topological insulators and superconductors, Rev. Mod. Phys. 83, 1057 (2011).

[44] M. Z. Hasan and C. L. Kane, Colloquium: Topological insulators, Rev. Mod. Phys. 82, 3045 (2010).

[45] B. A. Bernevig, T. L. Hughes, and S.-C. Zhang, Quantum spin Hall effect and topological phase transition in HgTe quantum wells, Science 314, 1757 (2006).

[46] H. Z. Lu, W. Y. Shan, W. Yao, Q. Niu, and S. Q. Shen, Massive Dirac fermions and spin physics in an ultrathin film of topological insulator, Phys. Rev. B 81, 115407 (2010).
[47] D. N. Sheng, Z. Y. Weng, L. Sheng, and F. D. M. Haldane, Quantum Spin-Hall Effect and Topologically Invariant Chern Numbers, Phys. Rev. Lett. 97, 036808 (2006).

[48] H. Li, L. Sheng, D. N. Sheng, and D. Y. Xing, Chern number of thin films of the topological insulator $\mathrm{Bi}_{2} \mathrm{Se}_{3}$, Phys. Rev. B 82, 165104 (2010).

[49] J. L. Collins, A. Tadich, W. Wu, L. C. Gomes, J. N. B. Rodrigues, C. Liu et al., Electric-field-tuned topological phase transition in ultrathin $\mathrm{Na}_{3} \mathrm{Bi}$, Nature (London) 564, 390 (2018).

[50] M. Kargarian, M. Randeria, and Y.-M. Lu, Are the surface Fermi arcs in Dirac semimetals topologically protected? Proc. Natl. Acad. Sci. USA 113, 8648 (2016).

[51] A. Roth, C. Brune, H. Buhmann, L. W. Molenkamp, J. Maciejko, X.-L. Qi, and S.-C. Zhang, Nonlocal transport in the quantum spin Hall state, Science 325, 294 (2009).

[52] R. Chen, S. Li, H.-P. Sun, Q. Liu, Y. Zhao, H.-Z. Lu, and X. C. Xie, Using nonlocal surface transport to identify the axion insulator, Phys. Rev. B 103, L241409 (2021).

[53] R. Landauer, Electrical resistance of disordered onedimensional lattices, Philos. Mag. 21, 863 (1970).

[54] M. Büttiker, Absence of backscattering in the quantum Hall effect in multiprobe conductors, Phys. Rev. B 38, 9375 (1988).

[55] D. S. Fisher and P. A. Lee, Relation between conductivity and transmission matrix, Phys. Rev. B 23, 6851 (1981).

[56] A. MacKinnon, The calculation of transport properties and density of states of disordered solids, Z. Phys. B: Condens. Matter 59, 385 (1985).

[57] G. Metalidis and P. Bruno, Green's function technique for studying electron flow in two-dimensional mesoscopic samples, Phys. Rev. B 72, 235304 (2005). 